\title{
Reaching the boundary between stellar kinematic groups and very wide binaries
}

\section{Sixteen new stars and eight new wide systems in the $\beta$ Pictoris moving group}

\author{
F. J. Alonso-Floriano ${ }^{1}$, J. A. Caballero ${ }^{2}$, M. Cortés-Contreras ${ }^{1}$, E. Solano ${ }^{2,3}$, and D. Montes ${ }^{1}$ \\ 1 Departamento de Astrofísica y Ciencias de la Atmósfera, Facultad de Ciencias Físicas, Universidad Complutense de Madrid, \\ 28040 Madrid, Spain \\ e-mail: fjalonso@ucm.es \\ 2 Centro de Astrobiología (CSIC-INTA), ESAC PO box 78, 28691 Villanueva de la Cañada, Madrid, Spain \\ 3 Spanish Virtual Observatory, ESAC PO box 78, 28691 Villanueva de la Cañada, Madrid, Spain
}

Received 19 June 2015 / Accepted 8 August 2015

\section{ABSTRACT}

\begin{abstract}
Aims. We look for common proper motion companions to stars of the nearby young $\beta$ Pictoris moving group.
Methods. First, we compiled a list of $185 \beta$ Pictoris members and candidate members from 35 representative works. Next, we used the Aladin and STILTS virtual observatory tools and the PPMXL proper motion and Washington Double Star catalogues to look for companion candidates. The resulting potential companions were subjects of a dedicated astro-photometric follow-up using public data from all-sky surveys. After discarding 67 sources by proper motion and 31 by colour-magnitude diagrams, we obtained a final list of 36 common proper motion systems. The binding energy of two of them is perhaps too small to be considered physically bound. Results. Of the 36 pairs and multiple systems, eight are new, 16 have only one stellar component previously classified as a $\beta$ Pictoris member, and three have secondaries at or below the hydrogen-burning limit. Sixteen stars are reported here for the first time as moving group members. The unexpected large number of high-order multiple systems, 12 triples and two quadruples among 36 systems, may suggest a biased list of members towards close binaries or an increment of the high-order-multiple fraction for very wide systems.
\end{abstract}

Key words. binaries: general - Galaxy: kinematics and dynamics - open clusters and associations: individual: $\beta$ Pictoris binaries: visual

\section{Introduction}

Wide binaries provide valuable information about key questions in astrophysics; for example, halo-wide pairs contribute to constraining the properties of dark matter (Weinberg et al. 1987; Yoo et al. 2004; Quinn et al. 2009), some star formation theories depend on the frequency and separation of wide young binaries (Parker et al. 2009; Ward-Duong et al. 2015; Marks et al. 2015), and relatively bright FGK-type primaries with M-dwarf companions provide a metallicity calibration yardstick for cool stars (Bonfils et al. 2005; Rojas-Ayala et al. 2012; Newton et al. 2014; Li et al. 2014). However, the maximum projected physical separation of a wide binary is still a matter of discussion: Some authors consider a cutoff in the number of wide binaries at $2 \times 10^{4}$ au $(\sim 0.1 \mathrm{pc})$, which is the typical size of protostellar cores (Tolbert 1964; Abt 1988; Wasserman \& Weinberg 1991; Allen et al. 2000; Tokovinin \& Lépine 2012), while others contemplate separations of $2 \times 10^{5}$ au $(\sim 1 \mathrm{pc})$ or more (Jiang \& Tremaine 2009; Caballero 2009; Shaya \& Olling 2011). Such wide common proper-motion pair candidates, which give their name to the title of this series of papers, can be either unbound members of the same young stellar kinematic group that by chance are co-moving (Tokovinin 2014a) or bound "binaries" of very low binding energies at the limit of disruption (Caballero 2010).

The younger a weakly bound system is, the less time it has had to be disrupted (Bahcall \& Soneira 1981; Retterer \& King 1982; Weinberg et al. 1987; Saarinen \& Gilmore 1989; Poveda $\&$ Allen 2004). As a result, a search for multiple systems within a young stellar kinematic group (moving group or stellar association) offers a unique opportunity for finding new faint benchmark objects hardly influenced by the Galactic gravitational potential, but instead by their formation process. In other words, the shape of young wide binaries is dominated by nature instead of by nurture.

In this work, we use the profitable technique of searching for common proper-motion pairs of wide separation (e.g., Luyten 1979; Chanamé \& Gould 2004) in a close and very young moving group, namely $\beta$ Pictoris (Zuckerman et al. 2001b; Ortega et al. 2002; Song et al. 2003). Although there is no consensus in the literature, the $\beta$ Pictoris age lies in a relatively narrow interval between $11 \mathrm{Ma}$ and 26 Ma (Barrado y Navascués 1998; Torres et al. 2006; Yee \& Jensen 2010; Binks \& Jeffries 2014; Mamajek \& Bell 2014, and references therein). Known moving group members and member candidates lie at between $6 \mathrm{pc}$ and $80 \mathrm{pc}$ from our Sun with a median distance of $40 \mathrm{pc}$.

Because of its youth and proximity, the $\beta$ Pictoris moving group has been relevant for studying resolved debris discs with high angular resolution observations (Smith \& Terrile 1984; Metchev et al. 2005; Boccaletti et al. 2009; Churcher et al. 2011; Wahhaj et al. 2013; Dent et al. 2013) and exoplanets through direct imaging (Mouillet et al. 1997; Neuhäuser et al. 2003; Kasper et al. 2007; Lagrange et al. 2009, 2010; Bonnefoy et al. 2011, 2013; Biller et al. 2013; Rameau et al. 2013; Males et al. 2014; Bowler et al. 2015; Macintosh et al. 2015) or for comparing observations with evolutionary models (Crifo et al. 1997; Song et al. 2002; Cruz et al. 2009; Biller et al. 2010; Mugrauer et al. 2010; Jenkins et al. 2012; Montet et al. 2015). Therefore, 
Table 1. Sources of the $\beta$ Pictoris stellar sample.

\begin{tabular}{|c|c|}
\hline Title & References \\
\hline Search for associations containing young stars (I, III, V, VI) & $\mathrm{SACY}^{a}$ \\
\hline Bayesian analysis to identify new star candidates in nearby young stellar... (I-V) & BANYAN $^{b}$ \\
\hline A dusty M5 binary in the $\beta$ Pictoris moving group & Rodríguez et al. (2014) \\
\hline On the age of the $\beta$ Pictoris moving group & Mamajek \& Bell (2014) \\
\hline The Solar Neighborhood. XXXIII. Parallax results from the CTIOPI $0.9 \mathrm{~m}$ program... & Riedel et al. (2014) \\
\hline A lithium depletion boundary age of $21 \mathrm{Myr}$ for $\beta$ Pictoris moving group & Binks \& Jeffries (2014) \\
\hline Unveiling new members in five nearby young moving groups & Moór et al. (2013) \\
\hline Identifying the young low-mass stars within $25 \mathrm{pc}$ (I, II) & Shkolnik et al. $(2009,2012)$ \\
\hline Likely members of the $\beta$ Pictoris and $\mathrm{AB}$ Doradus moving groups in the north & Schlieder et al. (2012b) \\
\hline Cool young stars in the northern hemisphere: $\beta$ Pictoris and AB Doradus moving... & Schlieder et al. (2012a) \\
\hline The sizes of the nearest young stars & McCarthy \& White (2012) \\
\hline Potential members of stellar kinematic groups within $30 \mathrm{pc}$ of the Sun & Nakajima \& Morino (2012) \\
\hline A search for new members of the $\beta$ Pictoris, Tucana-Horologium and $\eta$ Cha & Kiss et al. (2011) \\
\hline$\beta$ Pictoris and AB Doradus moving groups: likely new low-mass members & Schlieder et al. (2010) \\
\hline The lowest-mass member of the $\beta$ Pictoris moving group & Rice et al. (2010) \\
\hline Potential members of stellar kinematic groups within $20 \mathrm{pc}$ of the Sun & Nakajima et al. (2010) \\
\hline Kinematic analysis and membership status of TWA $22 \mathrm{AB}$ & Teixeira et al. (2009) \\
\hline Nearby young stars selected by proper motion. I. Four new members of the $\beta$ Pictoris... & Lépine \& Simon (2009) \\
\hline Young nearby loose associations & Torres et al. (2008) \\
\hline Unraveling the origins of nearby young stars & Makarov (2007) \\
\hline Nearby debris disk systems with high fractional luminosity reconsidered & Moór et al. (2006) \\
\hline Young stars near the Sun & Zuckerman \& Song (2004) \\
\hline New aspects of the formation of the $\beta$ Pictoris moving group & Ortega et al. (2004) \\
\hline New members of the TW Hydrae association, $\beta$ Pictoris moving group and Tucana... & Song et al. (2003) \\
\hline The origin of the $\beta$ Pictoris moving group & Ortega et al. (2002) \\
\hline The $\beta$ Pictoris moving group & Zuckerman et al. (2001b) \\
\hline The age of $\beta$ Pictoris & Barrado y Navascués et al. (1999) \\
\hline
\end{tabular}

Notes. ${ }^{(a)}$ SACY: Torres et al. (2006); da Silva et al. (2009); Elliott et al. (2014, 2015). ${ }^{(b)}$ BANYAN: Malo et al. (2013, 2014a,b); Gagné et al. (2014, 2015). We only collected BANYAN candidates with membership probability $P>50 \%$.

increasing the number of members via common proper-motion companionship, especially at low masses, can help to inform the previously mentioned fields and to constrain the age of the group. Besides that, identiying bright M-dwarf targets of $\sim 10$ $30 \mathrm{Ma}$ for extremely precise radial velocity surveys is becoming critical for understanding the formation and early evolution of terrestrial planets in habitable zones (Lissauer 2007; Ramírez \& Kaltenegger 2014; Luger et al. 2015; Tian 2015; Tian \& Ida 2015). Preliminary results of this work, including the discovery of two new stellar members in the $\beta$ Pictoris moving group, were given in Alonso-Floriano et al. (2011).

\section{Analysis}

\subsection{Stars sample}

We have compiled in Table A.1 a list of $185 \beta$ Pictoris members and member candidates around which we looked for common proper-motion companions. We gathered them from 35 previous works published in the past 16 years from the first articles of Barrado y Navascués et al. (1999) and Zuckerman et al. (2001b) to the last investigations published in the SACY (Search for Associations Containing Young stars - Torres et al. 2006; Elliott et al. 2014, 2015) and BANYAN series (Bayesian Analysis for Nearby Young AssociatioNs - Malo et al. 2014a,b; Gagné et al. 2015). Table 1 lists all works that we searched through.

We cross-matched our list with the latest GenevaCopenhagen catalogue (Holmberg et al. 2009) and identified 17 bright stars for which metallicity was available. From these data, we determined a solar metallicity of the $\beta$ Pictoris moving group of $[\mathrm{Fe} / \mathrm{H}]=-0.2 \pm 0.2$. In Table A.1, we provide for each star: discovery (or recommended) name, right ascension and declination from the Two-Micron All-Sky Survey (Skrutskie et al. 2006), heliocentric distance, its uncertainty when available, and corresponding reference. We follow the nomenclature convention of Alonso-Floriano et al. (2015). In particular, we provide for the first time the ROSAT precovery names (1RXS) for several stars for which no X-ray counterpart had been identified by subsequent proper-motion surveys.

In the last column, we also list a flag indicating the quality of the star membership in $\beta$ Pictoris:

1. Uncontrovertible moving group members for which at least two independent research groups have declared them to be bona fide moving group members and whose memberships have not been put in doubt afterwards. In general, these objects have coherent kinematics (with reliable distance and radial velocity determination) and youth features (coronal $\mathrm{X}$-ray and chromospheric $\mathrm{H} \alpha$ emission, lithium in absorption and, in some cases, debris discs).

2. Moving-group member candidates for which there is no definitive confirmation of true membership.

3. Dubious moving group member candidates that have also been proposed as belonging to other young moving groups of similar kinematics, or even to the field. We include them in our work for completeness.

\subsection{Proper motion companion candidates}

For this search, we made extensive use of virtual observatory tools. We used the comprehensive PPMXL proper motion 


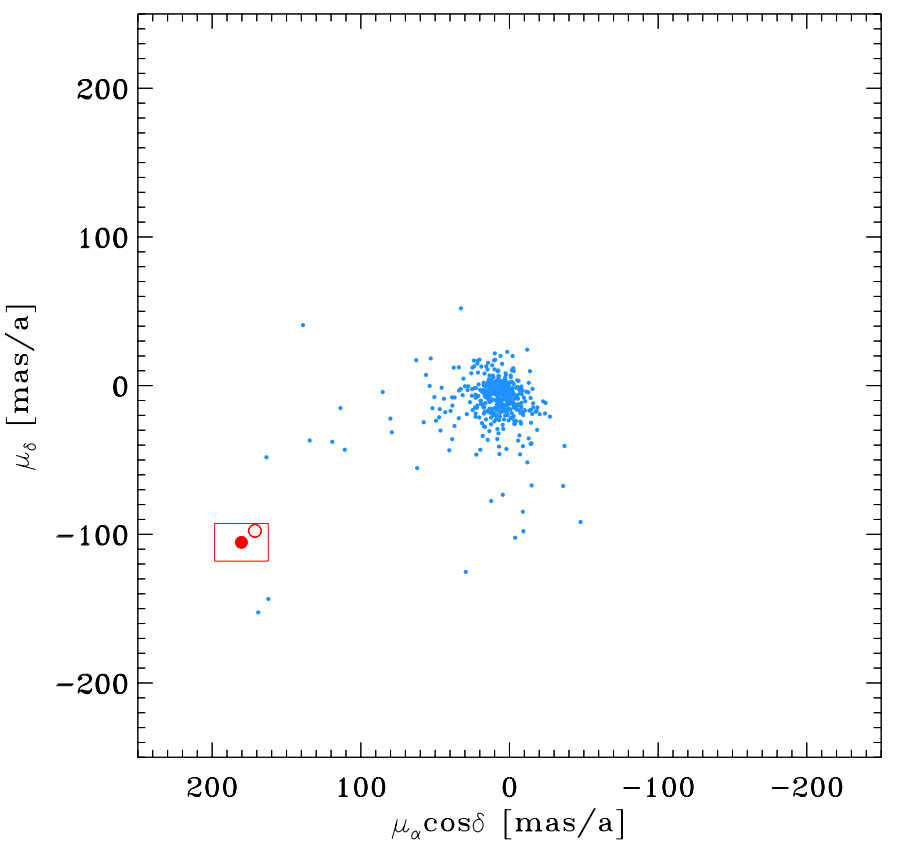

Fig. 1. Representative proper-motion diagram of all PPMXL sources brighter than $J=15.5$ mag in a 30 arcmin-radius circular area centred on LP 648-20. The red square box in the bottom left indicates the propermotion search area around LP 648-20, marked with a filled circle. The open circle corresponds to the bright, young G5 V star EX Cet.

catalogue (Roeser et al. 2010), the Aladin sky atlas (Bonnarel et al. 2000), and the Starlink Tables Infrastructure Library Tool Set (STILTS; Taylor 2006) to look for common proper-motion companions to the $185 \beta$ Pictoris stars in Table A.1. The PPMXL catalogue is complete down to the visual magnitude $V \approx 20 \mathrm{mag}$ and has typical individual mean errors of the proper motions between 4 and 10 mas/a, approximately. We applied the following selection criteria in our search.

- We looked for companion candidates in a circular area of angular radius $\rho=s / d$ (in arcsec) centred on each sample star, where $s$ is the maximum projected physical separation, fixed at $s=10^{5} \mathrm{au}$, and $d$ (in pc) is the heliocentric distance shown in Table A.1. At the given distances, the search radii varied between over $4 \mathrm{deg}$ for the closest $\beta$ Pictoris stars (e.g., $\mathrm{YZ} \mathrm{CMi} \mathrm{AB}$ at $5.96 \pm 0.08 \mathrm{pc}$ ) and 12 to $23 \mathrm{arcmin}$ for the most distant ones (e.g., LP 58-170 at $140 \pm 40$ pc and V4046 $\mathrm{Sgr} \mathrm{AB}$ and $\mathrm{C}$ at $73 \pm 18 \mathrm{pc}$ ). The median search radius was 44 arcmin.

- We discarded from the survey 24 stars with total PPMXL proper motions $\mu<50$ mas/a (19) or no proper motions at all (5). Therefore, we looked for companions of $161 \beta$ Pictoris stars. Stars slower than $\mu=50$ mas/a were not considered at this step because of the large number of potential candidates with relative uncertainties of $10 \%-30 \%$ in proper motion that would fall in the surveyed area and pass the filter. As proper motion companion candidates, we classified only the PPMXL sources with a 2MASS counterpart for which the values of $\mu_{\alpha} \cos \delta$ and $\mu_{\delta}$ lie within $10 \%$ of those of the primary target (see Fig. 1).

- We retained objects brighter than $J=15.5$ mag. In general, fainter sources in the near-infrared also have very faint magnitudes in the optical, close to the limit of the USNO-B1 (Monet et al. 2003) digitisations of $B_{J}, R_{F}$, and $I_{N}$ photographic plates, which were used by PPMXL. This faintness translates into large astrometric errors in the PPMXL proper motions. Keeping relatively bright sources assures the quality of the compiled astro-photometric measurements (see below), although prevents detecting fainter and, thus, low-mass $\beta$ Pictoris members in, perhaps, the substellar domain.

Once we had a preliminary list of candidates, we inspected all of them visually with Aladin and the images and data of various all-sky surveys (Palomar Observatory Sky Survey I and II; 2MASS; SDSS-DR9, Ahn et al. 2012; WISE, Cutri et al. 2012, 2014; CMC14 and CMC15, Evans et al. 2002). In particular, we checked that the companion candidates have a unique and reliable entry in the PPMXL catalogue (e.g., at least four astrometric detections, no other PPMXL source at less than 2 arcsec, smooth variation of the magnitudes from $B_{J}$, through $R_{J}, I_{N}, J, H, K_{\mathrm{s}}$, to WISE W1-4). In this step, we discarded a number of preliminary companion candidates with erroneous PPMXL proper motions (i.e., with incorrect USNO-B1 matches) owing to close visual multiplicity or source confusion in very crowded fields at low Galactic latitudes. Some of the mistaken sources were identified around $\alpha$ Cir, 1RXS J171502.4-333344, V4046 Sgr, 1RXS J184956.1-013402, which have $|b|<7$ deg, and, especially, V343 Nor, which is at less than 2 deg of the Galactic plane and, besides this, towards the Galactic centre. After this visual pre-cleaning, we obtained a list of 92 proper motion companion candidates to $65 \beta$ Pictoris stars.

Next, we performed a 10 arcsec-radius cross-match on our initial 185-star sample with the Washington Double Star catalogue (WDS - Mason et al. 2001, 2015). We got 163 positive cross-matches in 55 WDS systems. Of the cross-matches, 136 corresponded to close physical binaries not resolved by 2MASS nor PPMXL ( $\rho \lesssim 2.5$ arcsec) or to wider multiple systems, but with large magnitude differences measured with powerful adaptive optics systems (e.g., Lafrenière et al. 2007; Chauvin et al. 2010). The list of WDS systems unresolved or unidentified in our search are shown in Table A.2, which provides the star name, WDS discovery name (for resolved pairs) or reference (for spectroscopic binaries), multiplicity status (physical, visual - noncommon proper motion -, single/double-line spectroscopic binaries), angular separation (interval of $\rho$ when several visual companions are tabulated), position angle $(\theta)$, and WDS identifier (only for resolved pairs). For the physical and visual pairs in Table A.2, $\rho$ and $\theta$ correspond to the latest epoch listed by WDS.

Thanks to the cross-match with WDS, we were able to add another 15 previously known secondaries detected by 2MASS to our list of 92 proper motion companion candidates. They did not pass our filters above because they have PPMXL proper motions that deviate more than $10 \%$ from those of the "primary", probably because of relative orbital motion, erroneous measurements in right ascension and/or declination, proper motions with $1 \sigma$ lower limits below the 50 mas/a boundary, or no proper motions at all.

Finally, we also added to our list the companion candidates of three additional pairs of $\beta$ Pictoris stars that were not in WDS and that were not detected because of the reasons explained above: [SLS2012] PYC J02017+0117N \& S ( $\rho \sim 10$ arcsec and equal brightness) and TYC 112-917-1 \& $2 \mathrm{E} 1249 \mathrm{AB}(\mu \approx 41 \mathrm{mas} / \mathrm{a})$, which have the same predicted or measured distances and radial velocities (Schlieder et al. 2012a; Elliott et al. 2014) and are quite obvious proper motion companion candidates in Aladin, and V4046 Sgr AB and C, which was presented by Kastner et al. (2011).

In Table A.3, we list the $110(92+15+3)$ proper motion companion candidates that passed on to the next analysis stage. 

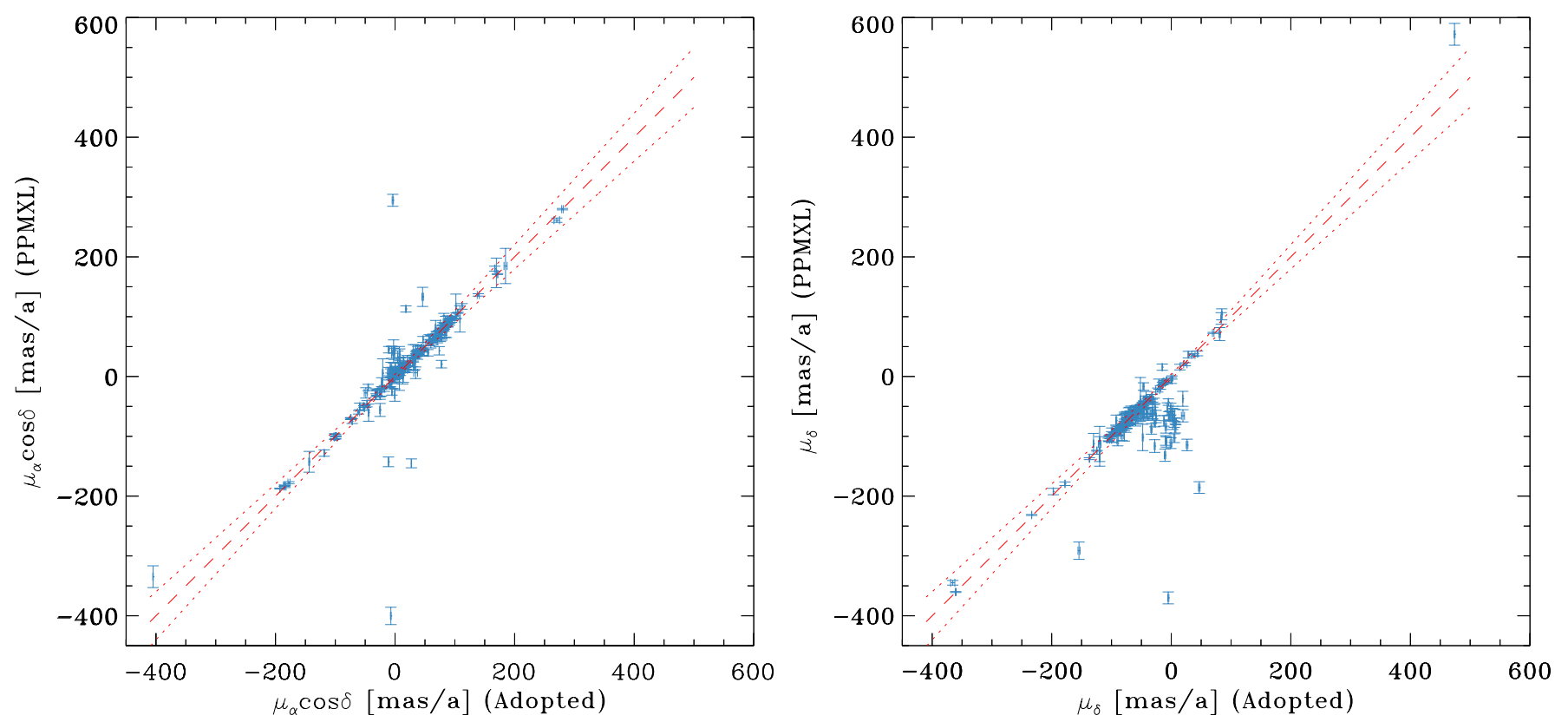

Fig. 2. PPMXL vs. adopted proper-motion diagrams in right ascension (left panel) and declination (right panel). The red dashed and dotted lines mark the one-to-one relationship and the $10 \%$ error area above and below it, respectively.

\subsection{Astro-photometric follow-up}

\subsubsection{Astrometry}

We performed a dedicated astro-photometric follow-up of the 110 companion candidates in two steps. In the first one, we confirmed true common proper motion of the pairs with a precise astrometric study. This step was necessary because PPMXL used the astro-photometric USNO-B1 catalogue as input, which is known to be affected by systematics at the fainter optical magnitudes, especially when dealing with high proper motion stars.

Of the 184 objects in Table A.3 (74 primaries and 110 companion candidates), 55 had reliable proper motions measured by HIPPARCOS (TYC, Høg et al. 2000; HIP2, van Leeuwen 2007). For the remaining 129 objects, we measured precise proper motions from public data in virtual observatory catalogues as in Caballero $(2010,2012)$. In particular, we used astrometric epochs from the following catalogues: AC2000.2 (Urban et al. 1998), USNO-A2 (Monet 1998), GSC2.3 (Lasker et al. 2008), DENIS (Epchtein et al. 1997), CMC14 and CMC15, 2MASS, SDSS, and WISE. To maximise the number of astrometric epochs, $N$, and time baseline, $\Delta t$, of the follow-up, which translates into reducing the uncertainty in proper motion, we also used the SuperCOSMOS digitisations of Palomar Observatory Sky Survey photographic plates, especially for the faintest objects (Hambly et al. 2001; cf., Caballero 2012). The addition of SuperCOSMOS data allowed us to get at least four accurate astrometric epochs spread over a minimum of $11.5 \mathrm{a}$ for all targets except for one star (2MASS J05113065-2155189, $N=3$ ). The average number of astrometric epochs and time baseline were five and $34 \mathrm{a}$, respectively. In the extreme case of TYC 45711414-1, we measured its proper motion with eight astrometric epochs spread over almost 115 a. Table A.3 lists the 2MASS coordinates of the 184 "primaries" and companion candidates, and their PPMXL and adopted proper motions. For the adopted proper motions that do not come from TYC or HIP2, Table A.3 also provides the time baseline and number of epochs used in our astrometric follow-up. Except for partially resolved close binaries (e.g., AT Mic AB) or faint sources ( $r^{\prime} \gtrsim 16 \mathrm{mag}$ ), we were able to measure proper motions with typical uncertainties of 1 mas/a or less, which are comparable to or even better than TYC or HIP2.

We show a comparison of the original PPMXL proper motion values and the ones adopted by us in Fig. 2. While the values of proper motions in right ascension provided by PPMXL have in general good agreement with our adopted values, many PPMXL proper motions in declination have greater absolute values.

With the new data, we made a second, more precise, astrometric filtering and discarded 43 visual companions with adopted proper motions that deviate more than $10 \%$ from the proper motion of the system. This step of the follow-up thus left 67 physical companion candidates for the second step. In general, the astrometrically rejected objects are distant background stars with fake high proper motions in the PPMXL catalogue, which are located close to bright stars and were reported previously as companion candidates or which are located at large angular separations to our primary targets and have by chance similar, but not identical, proper motions. Of the rejected stars, four were catalogued companion candidates of c Eri, $\alpha$ Cir, CD-24 16238, and AF Psc, and one was a faint companion candidate to [SLS2012] PYC J10175+5542 (Schlieder et al. 2012a) that had been reported by W. J. Luyten (LDS 2851, WDS 10176+5542).

\subsubsection{Photometry}

In the second step of the follow-up, we studied the membership in the $\beta$ Pictoris moving group of the 67 companion candidates that passed the previous astrometric filter with the help of colourmagnitude diagrams and theoretical isochrones.

First, we compiled $B, V, r^{\prime}, J, H, K_{\mathrm{s}}$, and $W 1-4$ magnitudes for all the sources investigated in this work. While infrared $J H K_{\mathrm{S}} W 1-4$ photometry came in all cases from 2MASS and WISE, the origin of the optical $B V r^{\prime}$ photometry was diverse. When available, we collected $B V r^{\prime}$ photometry from UCAC4 (Zacharias et al. 2013). If not available, we got it from a number 
F. J. Alonso-Floriano et al.: Sixteen new stars and eight new wide systems in the $\beta$ Pictoris moving group
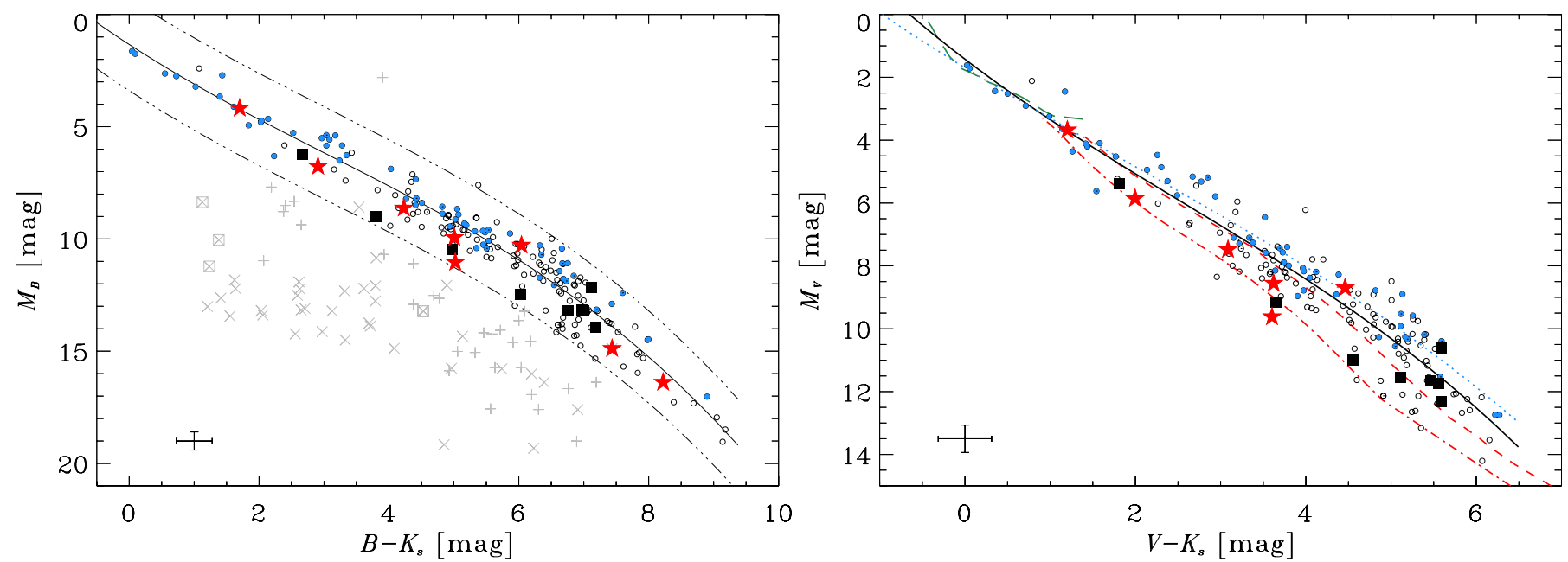

Fig. 3. Optical-to-near-infrared colour-magnitude diagrams of our $\beta$ Pictoris stars and common proper-motion companion candidates. In both panels, blue filled circles mark bona fide moving group members with membership flag 1 in Table A.1 (see Sect. 2.1), and open circles indicate other member candidates with flags 2 and 3. Black filled squares denote companions previously known in the literature that had not been reported as belonging to $\beta$ Pictoris. Red filled stars mark our eight new proper motion companions. Typical error bars are shown in the bottom left corner. The black solid line is the average $\beta$ Pictoris sequence computed with all candidate members as in the left panel (flags 1,2, and 3). Left panel: $M_{B}$ vs. $B-K_{\mathrm{s}}$ diagram. The dash-dotted lines are the $\beta$ Pictoris sequence shifted by $\pm 3 \sigma$. Grey times $(\times)$ and crosses $(+)$ indicate discarded companion candidates from astrometry and photometry, respectively; squared symbols mark companion candidates in the WDS. Right panel: $M_{V}$ vs. $V-K_{\mathrm{s}}$ diagram. The blue dotted line is the sequence with only bona fide members (flag 1). Red dashed and dash-dotted lines are the 20 and $100 \mathrm{Ma}$ isochrones from Baraffe et al. (2015). Green long dashed line is the $20 \mathrm{Ma}$ isochrone from Siess et al. (2000) plotted only at highest masses. For clarity, we do not draw the discarded companion candidates. Some remarkable stars do not have $V$ photometry.

of sources: Tycho-2 ( $B$ and $V$, after transformation from $B_{\mathrm{T}}$ and $V_{\mathrm{T}}$ magnitudes), USNO-B1 ( $B$, after average of two photographic $B_{J}$ magnitudes), AC2000.2 (only one star), SPM4 (Girard et al. $2011-V$, only one star), CMC $15\left(r^{\prime}\right)$, SDSSDR9 $\left(r^{\prime}\right)$, or the literature (Voges et al. 1999; Bakos et al. 2002; Torres et al. 2006; Beichman et al. 2010; Smart 2013).

We were not able to compile optical $B V r^{\prime}$ photometry for all our targets. Since we were able to compile magnitudes for more stars in the $B$ band, we applied our photometric filtering using the reddest near-infrared band, $K_{\mathrm{s}}$, and the bluest optical one, $B$. Actually, we failed to find reliable $B$ photometry for only six stars: five low-mass stars or brown-dwarf candidates with spectral types at the M/L boundary, of which four are from Gagné et al. (2014, 2015), one is the known companion of L 186-67 A (see below), and the sixth one is a star close to the bright primary V343 Nor A. The reddest and faintest object in our sample with $B$ and $K_{\mathrm{s}}$ photometry is 2MASS J06085283-2753583 (M8.5 V, Luhman et al. 2009; $B-K_{\mathrm{s}}=9.1 \mathrm{mag}$ ). The use of WISE photometry did not provide significant improvement over the use of 2MASS $K_{\mathrm{s}}$.

We performed our photometric filtering in a recursive scheme:

- First we computed the $B$-band absolute magnitude $M_{B}$ with the heliocentric distances in Table A.1 and built the $M_{B}$ vs. $B-K_{\mathrm{S}}$ diagram in left-hand panel of Fig. 3. Any new companion candidate would have to be located at the same distance as the target star.

- We defined an average $\beta$ Pictoris sequence with all members and candidates in Table A.1 (flags 1, 2, and 3). All sources that did not pass the astrometric filter lie in the locus of background stars in the colour-magnitude diagram.

- We checked the reliability of our average sequence by comparing it with the latest evolutionary models by Baraffe et al. (2015). Since BT-Settl does not provide $M_{B}$ magnitudes, we used $M_{V}$ ones instead. We built the $M_{V}$ vs. $V-K_{\mathrm{s}}$ diagram in right-hand panel of Fig. 3 and plotted the corresponding
BT-Settl 20 and $100 \mathrm{Ma}$ isochrones. The acceptable match between our average $M_{V}$ vs. $V-K_{\mathrm{s}}$ sequence and the $20 \mathrm{Ma}$ isochrone encouraged us to use our average $M_{B}$ vs. $B-K_{\mathrm{s}}$ sequence for the photometric filter.

- We discarded stars with absolute magnitudes $M_{B}$ and colours $B-K_{\mathrm{s}}$ inconsistent with the $\beta$ Pictoris sequence. Most discarded stars lie outside the $\pm 3 \sigma$ area around the sequence. The systematic error introduced by mixing different photometric systems for the blue magnitude seems to be smaller than the intrinsic scatter in the $\beta$ Pictoris sequence, mostly due to uncertainties in distance.

Of the previous 67 stars, 31 did not pass the photometric filter. One known system, L 186-67 Aa, Ab, B, could not be studied photometrically because of the lack of reliable data in the optical, but the short angular separation between components and the large common proper motions ensured that it is a physical system.

\section{Results and discussion}

\subsection{Known and new common proper motion pairs}

From the initial list of 184 common proper motion companion candidates to $\beta$ Pictoris stars in Sect. 2.2, only 36 targets passed the two filters of our astro-photometric follow-up in Sect. 2.3. Our final list of confirmed common-proper motion systems in the $\beta$ Pictoris moving group, as shown in Table A.5, consists of

- Eighteen known systems in which the two stars had been reported previously to belong to the moving group. All of them except one are listed by WDS; the exception is the wide system formed by V4046 Sgr AB and V4046 Sgr C, which was proposed and investigated for the first time by Kastner et al. (2011). Some of the 17 WDS systems have been known for decades, such as five pairs in the W. J. Luyten's Double Star catalogue or the HD 14082 AB pair, which was resolved for the first time by F. G. W. Struve in 1821 . 
- Ten known systems in which only one star had been reported previously as belonging to the moving group. Of the ten stars that had not been reported as belonging to $\beta$ Pictoris (i.e., not listed in Table A.1), four displayed significant X-ray emission in ROSAT observations (Voges et al. 1999; Riaz et al. 2006; Kaplan et al. 2006; Haakonsen \& Rutledge 2009) and two showed intense $\mathrm{H} \alpha$ emission at the chromospheric/accretion boundary for their spectral types $(p E W(\mathrm{H} \alpha) \approx-12$ to $-16 \AA$ - Reid et al. 1995; Riaz et al. 2006). Since there is one star that is an $\mathrm{H} \alpha$ and $\mathrm{X}$-ray emitter (2MASS J00193931+1951050), half of the ten new stars have known signposts of youth, which supports membership in $\beta$ Pictoris. Besides this, another one has a similar radial velocity to the primary in the system (CD-44 753 A and B Kordopatis et al. 2013). For the other four new young stars, there are only photometric data available (and, in the case of 2MASS J07293670+3554531, mass and spectral type derived from photometry - Pickles \& Depagne 2010; Janson et al. 2012).

- Eight new common proper motion systems with $\beta$ Pictoris stars. In reality, there are WDS entries for two $\beta$ Pictoris pairs that were presented for the first time by Alonso-Floriano et al. (2011): EX Cet A, B (CAB 3) and HD 173167 A, B (CAB 8). Although the results from this preliminary publication have already been used by other authors (Shkolnik et al. 2012; Eisenbeiss et al. 2013; Bowler et al. 2015), we consider their discovery as part of this work. Moór et al. (2013) "rediscovered" the pair HD 173167 A, B, although they did not report $\rho$ or $\theta$. The optical spectra of these two stars and of TYC 112-917-1 and 2E 1249 AB in the new pair WDS 05200+0613 display intense Li I $16707.8 \AA$ line in absorption for their spectral type (Alcalá et al. 2000; Torres et al. 2006), which supports their extremely young age. Six of these stars are reported here as new member candidates in the $\beta$ Pictoris moving group.

On some occasions we use the term "pair" to refer to multiple systems that contain only two components resolvable from the ground with standard imaging (i.e., no adaptive optics or lucky imaging) and spectroscopic devices. Most of our systems are such pairs. However, Table A.5 lists 12 triple and two quadruple hierarchical systems that contain one or two close pairs unresolved by public catalogues (Table A.2). The two quadruple systems are MV Vir Aa, Ab, B, C and HD 199143 AB, CD (for which the close components were resolved first by Jayawardhana \& Brandeker 2001). The latter has an "A. Tokovinin" WDS entry dated after 2011, but the wide multiplicity was previously reported by Alonso-Floriano et al. (2011) and, especially, Zuckerman et al. (2001b).

The existence of 14 triples and quadruples in a list of $36 \mathrm{mul}-$ tiples provides a high-order-multiple ratio of about 1:3, which is unexpectedly high. Law et al. (2010) found a similar ratio of about 1:2 for wide M-dwarf binaries of the field and suggest that some of the binaries with large separations are actually triple and quadruple systems. (Actually, Caballero 2007 and Burgasser et al. 2007 pointed it out before.) The increment of the high-order-multiple fraction for the widest systems is supported by the work of Reipurth \& Mikkola (2012), who used $N$-body simulations of the dynamical evolution of triple systems to suggest that loosely bound triple systems might appear to be very wide binaries. However, recent dedicated surveys for multiplicity of F, G, K (Tokovinin et al. 2014b; Elliott et al. 2015) and M dwarfs in the field (Cortés-Contreras et al. 2014) have found lower ratios of about 1:10. Although our sample comprises a wide range of masses and separations, it is not large enough to do an appropriate comparison with the previously mentioned works. Another explanation might be an observational effect of a biased sample in which surveys for nearby young stars are naturally slanted towards detecting intrinsically bright binaries and multiple stars (Malmquist bias), and active spectroscopic binaries (very close separations enhances stellar activity). The discovery of new moving groups members only based on astrometry, as in this survey, may help to alleviate this observational bias.

In Table A.5 we list our WDS identifiers in italics if they are not included in the WDS catalogue at the time of writing these lines (i.e., V4046 Sgr AB,C and six of the eight new pairs). In total, in this survey we propose 16 new stellar members of the $\beta$ Pictoris moving group: six in new pairs and ten in known systems with only one reported young star. One of the new $\beta$ Pictoris stars in a new pair is HD $173167 \mathrm{~A}$, which was discovered by Alonso-Floriano et al. (2011) and classified afterwards as a moving group member by Moór et al. (2013). These values represent an increase of $9 \%$ in the total number of reported $\beta$ Pictoris stars and of almost $30 \%$ in the number of wide proper motion systems in the moving group. We ran the on-line BANYAN tool ${ }^{1}$ (Malo et al. 2013) on the 16 new proposed members of $\beta$ Pictoris and calculated approximate membership probabilities (Table 2). We used the distances of the systems provided in Table A.5, our proper motion measurements in Table A.3, and radial velocities from the literature (for those objects without radial velocity measurements, we assumed the values of their companions). Although only seven of the 16 pairs showed high-probability memberships to $\beta$ Pictoris (see Table 2), these results should be used with caution because most of the new candidates lack accurate distances or radial velocities.

None of the new reported wide systems have parallax measurements for both components. However, the location of the 16 stars (eight primaries and eight secondaries) in the colourmagnitude diagrams suggests that both components are located at similar distances. Definitive parallactic confirmation of common distance will have to wait until early 2017 with the second Gaia release. In the meantime, we can infer the true physical binding of the systems with the computation of the reduced gravitational binding energy.

\subsection{Projected separations and binding energies}

In Table A.5 we list the angular separations, $\rho$, and position angles, $\theta$, at the 2MASS epoch of observation of the 36 wide pairs in the $\beta$ Pictoris moving group. Angular separations vary from 8.2 arcsec for BD-21 1074A, Ba, Bb to about $1.3 \mathrm{deg}$ for the triple system AU Mic-AT Mic AB (Luyten 1941; Caballero 2009). Among our new pairs, $\rho$ varies from $10.6 \operatorname{arcsec}$ to 24.5 arcmin.

To distinguish between true very wide physical binaries and co-moving pairs of "single" stars that belong to the same kinematic group, we computed the reduced gravitational binding energies, $U_{\mathrm{g}}^{*}=-G \mathcal{M}_{1} \mathcal{M}_{2} s^{-1}$ (Caballero 2009), of the 36 systems. With the angular separations and distances, we obtained the projected physical separations, $s$, which vary from merely 100-120 au for the known pairs WDS 08228-5727 (L 186$67 \mathrm{Aa}, \mathrm{Ab}, \mathrm{B}$ ) and WDS 10596+2527 (HD $95174 \mathrm{AB}$ ) to about $7 \times 10^{4}$ au $(0.34 \mathrm{pc})$ for the new pair WDS $08290+1125$. Given the uncertainties in the distance (Table A.1), we provide only two significant figures for $s$.

http://www . astro. umontreal.ca/ malo/banyan.php 
Table 2. Membership probabilities for the 16 new $\beta$ Pictoris candidates using the BANYAN on-line tool.

\begin{tabular}{|c|c|c|c|c|}
\hline $\begin{array}{l}\text { Simbad } \\
\text { name }\end{array}$ & $\begin{array}{c}\beta \text { Pic } \\
\text { prob. [\%] }\end{array}$ & $\begin{array}{l}\text { Highest } \\
\text { prob. [\%] }\end{array}$ & $\begin{array}{c}V_{\mathrm{r}} \\
{\left[\mathrm{km} \mathrm{s}^{-1}\right]}\end{array}$ & $\begin{array}{l}\text { Reference } \\
\text { of } V_{\mathrm{r}}\end{array}$ \\
\hline 2MASS J00193931+1951050 & 83.9 & $\beta$ Pic & $-1.7 \pm 1.0^{P}$ & Schlieder et al. (2012a) \\
\hline EX Cet & 99.9 & $\beta$ Pic & $+41.8 \pm 0.7$ & Soubiran et al. (2013) \\
\hline CD-44 753 B & 0.1 & Tuc-Hor (99.4) & $+12.4 \pm 1.9$ & Kordopatis et al. (2013) \\
\hline 2MASS J07293670+3554531 & 23.4 & Field (50.8) & $+10.4 \pm 0.9^{P}$ & Schlieder et al. (2012a) \\
\hline L 186-67 B & 0.0 & Field (100) & $+40 \pm 9^{P}$ & Kordopatis et al. (2013) \\
\hline 2MASS J08274412+1122029 & 16.0 & Field (84.0) & $+11.2 \pm 1.7^{P}$ & Schlieder et al. (2012a) \\
\hline HD 82939 A & 1.1 & Field (98.9) & $-0.5 \pm 0.4$ & Gontcharov (2006) \\
\hline 2MASS J12120849+1248050 & 0.0 & Field (99.9) & $-4.0 \pm 1.0^{P}$ & Schlieder et al. (2012a) \\
\hline MV Vir C & 0.0 & AB Dor (86.0) & $+0.0 \pm 0.8^{P}$ & Malo et al. (2014a) \\
\hline 2MASS J16170673+7734028 & 0.0 & Field (100) & $-14.4 \pm 1.0^{P}$ & Schlieder et al. (2012a) \\
\hline 2MASS J18420483-5554126 & 99.9 & $\beta$ Pic & $+1.0 \pm 0.7^{P}$ & Malo et al. (2013) \\
\hline HD 173167 A & 99.9 & $\beta$ Pic & $+0.8 \pm 7.0$ & Moór et al. 2013 \\
\hline HDE 331149 B & 95.3 & $\beta \mathrm{Pic}$ & $-19.2 \pm 1.1^{P}$ & Schlieder et al. (2012a) \\
\hline BPS CS 22898-0066 & 0.0 & Field (100) & $+0.6 \pm 3.0$ & Kordopatis et al. (2013) \\
\hline 2MASS J21551738-0046231 & 75.2 & $\beta$ Pic & & \\
\hline 2MASS J23301129-0237227 & 91.2 & $\beta$ Pic & $-5.3 \pm 0.2^{P}$ & Malo et al. (2014b) \\
\hline
\end{tabular}

Notes. ${ }^{(P)}$ Radial velocity adopted from the primary component.

We derived masses $\mathcal{M}_{1}$ and $\mathcal{M}_{2}$ from $J$-band absolute magnitudes $M_{J}$ and the Baraffe et al. (2015) or Siess et al. (2000) evolutionary models at $20 \mathrm{Ma}$ for solar metallicity and the appropriate mass intervals. When available, we gathered masses of single early type stars and close binaries from the literature (e.g., Strassmeier \& Rice 2000; Neuhäuser et al. 2002; Caballero 2009; Donati et al. 2011; Janson et al. 2012; Elliott et al. 2015; Montet et al. 2015) or suitable information that allowed us to make a precise derivation (e.g., magnitude differences from adaptive optics or lucky imaging, mass ratios from spectroscopic monitoring - Chauvin et al. 2010; Neuhäuser et al. 2011; Janson et al. 2012; Messina et al. 2014; Bowler et al. 2015; Elliott et al. 2015). Masses range approximately from $2.4 M_{\odot}$ for $\eta$ Tel A to well below the substellar boundary for L 186$67 \mathrm{~B}$ with a broad maximum of the distribution at $0.5-1.0 M_{\odot}$. Derived masses reasonably match those expected from spectral types, when available. For the sake of completeness, we also list spectral types compiled from a number of sources in Table A.5 (Riaz et al. 2006; Reid et al. 2007; Pickles \& Depagne 2010; Caballero 2012; Janson et al. 2012; Kraus et al. 2014; Messina et al. 2014; Rodríguez et al. 2014; Mason et al. 2015; I. Gallardo \& M. Gómez Garrido, priv. comm.; SIMBAD).

The greatest absolute value of reduced binding energy among the 36 systems in Table A.5, of $-U_{\mathrm{g}}^{*}=9800 \times 10^{33} \mathrm{~J}$, corresponds to the strongly bound pair HD $95174 \mathrm{AB}$, which is not only the tightest one, but also contains two stars of $\sim 0.8 M_{\odot}$. On the other hand, there are two very fragile system candidates with binding energies of $0.57-2.710^{33} \mathrm{~J}$, almost one order of magnitude lower than that of the Luyten's system AU Mic+AT Mic AB, which lies at the boundary between very wide binaries and couples of single stars that are co-moving within the same stellar kinematic group (Caballero 2010; see the title of this series of papers). As a result, it is likely that the components in the two new fragile system candidates WDS $08290+1125$ and WDS 23317-0245, which includes the flaring star AF Psc (Bond 1976; Kraus et al. 2014; Ramsay \& Doyle 2014), originated in the same parental cloud and were ejected at the same time, in the same direction, and at the same velocity, but they are not physically bound. The six other new pairs have binding energies between 13 and $1400 \times 10^{33} \mathrm{~J}$ and may survive the eventual disruption by the Galactic gravitational potential for some billion years (Weinberg et al. 1987; Close et al. 2007). In any case, detecting features of youth in the spectra of WDS $08290+1125 \mathrm{~A}$ and B and the wide M6.0 V companion candidate to AF Psc (Reid et al. 2007) would shed light on their actual membership in the $\beta$ Pictoris moving group.

\subsection{Benchmark objects and probable members in other young moving groups}

The 36 wide systems tabulated by us can help to constrain the actual membership of some controversial candidate members in $\beta$ Pictoris:

- WDS 01367-0645. Some authors have also classified the primary of the system, EX Cet (G5V), as a member of the Hercules-Lyra association ( $100-200 \mathrm{Ma}-$ Montes et al. 2001; López-Santiago et al. 2006; Shkolnik et al. 2012; Eisenbeiss et al. 2013).

- WDS 02305-4342. The primary CD-44 $753 \mathrm{~A}$ is also a member candidate of the Columba association $(\sim 15-50 \mathrm{Ma}$ - Torres et al. 2006, 2008; Elliott et al. 2014; Malo et al. 2014a).

- WDS 08228-5727. The membership of the primary L 186-67 Aa, Ab to $\beta$ Pictoris is ambiguous (Malo et al. 2013 , 2014a). The late-M common proper motion companion, L 186-67 B, whose physical binding in the system had been confirmed earlier (Bakos et al. 2002; Bergfors et al. 2010; Janson et al. 2012, 2014), would have a mass close to the deuterium-burning mass limit if it were $20 \mathrm{Ma}$ old. If membership in $\beta$ Pictoris were confirmed, the triple system would be a benchmark for very low-mass substellar astrophysics.

- WDS 09361+3733. While there are no membership studies for the primary, the homonymous secondary HD $82939 \mathrm{Ba}, \mathrm{Bb}$ was listed not only as a $\beta$ Pictoris star by Schlieder et al. (2012a,b), but also as a young field star by Malo et al. (2014b).

- WDS 16172+7734. Schlieder et al. (2012a) listed the primary TYC 4571-1414-1 as a probable member of both $\beta$ Pictoris and $\mathrm{AB}$ Doradus ( $\sim 70 \mathrm{Ma})$ moving groups. 
- WDS 21214-6655. The primary star V390 Pav A has also been classified as a member of the Tucana-Horologium association ( $30 \mathrm{Ma}$ - Zuckerman et al. 2001a; Mamajek et al. 2004; Rojas et al. 2008).

If the six systems above were eventually discarded as true $\beta$ Pictoris "pairs", 30 systems would still remain for further investigation in the young moving group, of which six (20\%) are reported here for the first time.

Certain systems in Table A.5 are also particularly important in the low-mass domain, because they can be used to test evolutionary models. Just to cite one example, the secondary of the pairs WDS $16172+7734$ (presented here for the first time) and WDS 21105-2711 (Bergfors et al. 2010; Malo et al. 2013, 2014b) lie close to the substellar limit and, therefore, to the lithium depletion boundary. As a result, a high-resolution spectroscopic analysis of both primaries and secondaries could shed more light on the debated age of $\beta$ Pictoris.

\section{Conclusions}

We searched through 35 previous publications and compiled an exhaustive list of 185 members and member candidates in the nearby, young ( 20 Ma) $\beta$ Pictoris moving group, around which we looked for common proper-motion companions at projected physical separations of up to $10^{5}$ au. For that, we made extensive use of the Aladin and STILTS virtual observatory tools and numerous public all-sky catalogues (e.g., WDS, PPMXL, 2MASS).

Of the 184 initial common proper-motion companion candidates, 129 were the subject of a precise astrometric follow-up, by which we measured proper motions with typical uncertainties of only 1 mas/a, and 67 of a multi-band photometric study. Eventually, we discarded five previously reported pairs and retained 36 reliable pair candidates. Of them, 18 and 10 are known systems with both components or only one component classified as $\beta$ Pictoris members, respectively, and eight are new pairs in the moving group. We also report 16 new star and brown dwarf candidates in $\beta$ Pictoris for the first time. These values represent an increase of $9 \%$ in the total number of reported objects in the moving group and of almost $30 \%$ in the number of wide proper motion systems.

We investigated the 36 pairs with available public information in detail. Among them, there are 12 triple and two quadruple systems, which points out to a greater incidence of high-order multiplicity in $\beta$ Pictoris than in the field, possibly ascribed to a member list biased towards close binaries or an increment of the high-order, multiple fraction for very wide systems.

We measured angular separations and projected physical separations, compiled or derived masses for components in all systems, and computed reduced gravitational binding energies. Two of the new pair candidates could be unbound couples of single stars that are co-moving within $\beta$ Pictoris, while at least one of the components in six (new and known) pairs have also been reported to belong to other young moving groups and associations (four in Hercules-Lyra, Columba, AB Doradus, TucanaHorologium) or to the field (two). There are three pairs (one presented here) with masses of secondaries at or below the hydrogen-burning limit, and they can be used as benchmarks for upcoming age-dating works in $\beta$ Pictoris. Our study provides a comprehensive analysis of the wide multiplicity in one of the closest and youngest moving groups known and, therefore, also serves as input to models of moving-group evolution and eventual dissipation by the Galactic gravitational field.
Acknowledgements. We thank the anonymous referee for the report. This research made use of the Washington Double Star Catalogue, maintained at the US Naval Observatory, the SIMBAD database and VizieR catalogue access tool, operated at Centre de Données astronomiques de Strasbourg, France, and the Spanish Virtual Observatory (http://svo.cab.inta-csic. es). Financial support was provided by the Universidad Complutense de Madrid, the Comunidad Autónoma de Madrid, and the Spanish Ministerios de Ciencia e Innovación and of Economía y Competitividad under grants AP2009-0187, AYA2011-24052, and AYA2011-30147-C03-02, and -03.

\section{References}

Abt, H. A. 1988, Ap\&SS, 142, 111

Ahn, C. P., Alexandroff, R., Allende Prieto, C., et al. 2012, ApJS, 203, 21

Alcalá, J. M., Covino, E., Torres, G., et al. 2000, A\&A, 353, 186

Allen, C., Poveda, A., \& Herrera, M. A. 2000, A\&A, 356, 529

Alonso-Floriano, F. J., Caballero, J. A., \& Montes, D. 2011, in Proc. Stellar Clusters \& Associations: A RIA Workshop on Gaia, 344

Alonso-Floriano, F. J., Morales, J. C., Caballero, J. A., et al. 2015, A\&A, 577, A128

Bahcall, J. N., \& Soneira, R. M. 1981, ApJ, 246, 122

Bakos, G. Á., Sahu, K. C., \& Németh, P. 2002, ApJS, 141, 187

Baraffe, I., Homeier, D., Allard, F., \& Chabrier, G. 2015, A\&A, 577, A42

Barrado y Navascués, D. 1998, Ap\&SS, 263, 235

Barrado y Navascués, D., Stauffer, J. R., Song, I., \& Caillault, J.-P. 1999, ApJ, 520, L123

Beichman, C. A., Krist, J., Trauger, J. T., et al. 2010, PASP, 122, 162

Bergfors, C., Brandner, W., Janson, M., et al. 2010, A\&A, 520, A54

Biller, B. A., Liu, M. C., Wahhaj, Z., et al. 2010, ApJ, 720, L82

Biller, B. A., Liu, M. C., Wahhaj, Z., et al. 2013, ApJ, 777, 160

Binks, A. S., \& Jeffries, R. D. 2014, MNRAS, 438, L11

Boccaletti, A., Augereau, J.-C., Baudoz, P., Pantin, E., \& Lagrange, A.-M. 2009, A\&A, 495, 523

Bond, H. E. 1976, IBVS, 1160, 1

Bonfils, X., Delfosse, X., Udry, S., et al. 2005, A\&A, 442, 635

Bonnarel, F., Fernique, P., Bienaymé, O., et al. 2000, A\&AS, 143, 33

Bonnefoy, M., Lagrange, A.-M., Boccaletti, A., et al. 2011, A\&A, 528, L15

Bonnefoy, M., Boccaletti, A., Lagrange, A.-M., et al. 2013, A\&A, 555, A107

Bowler, B. P., Liu, M. C., Shkolnik, E. L., \& Tamura, M. 2015, ApJS, 216, 7

Burgasser, A. J., Reid, I. N., Siegler, N., et al. 2007, Protostars and Planets V, 427

Caballero, J. A. 2007, A\&A, 462, L61

Caballero, J. A. 2009, A\&A, 507, 251

Caballero, J. A. 2010, A\&A, 514, A98

Caballero, J. A. 2012, The Observatory, 132, 1

Caballero, R. 2012, J. Double Star Observations, 8, 58

Chanamé, J., \& Gould, A. 2004, ApJ, 601, 289

Chauvin, G., Lagrange, A.-M., Bonavita, M., et al. 2010, A\&A, 509, A52

Churcher, L., Wyatt, M., \& Smith, R. 2011, MNRAS, 410, 2

Close, L. M., Zuckerman, B., Song, I., et al. 2007, ApJ, 660, 1492

Cortés-Contreras, M., Caballero, J. A., \& Montes, D. 2014, The Observatory, 134,348

Crifo, F., Vidal-Madjar, A., Lallement, R., Ferlet, R., \& Gerbaldi, M. 1997, A\&A, 320, L29

Cruz, K. L., Kirkpatrick, J. D., \& Burgasser, A. J. 2009, AJ, 137, 3345

Cutri, R. M. et al. 2012, VizieR on-line catalogue: II/311

Cutri, R. M. et al. 2014, VizieR on-line catalogue: II/328

da Silva, L., Torres, C. A. O., de La Reza, R., et al. 2009, A\&A, 508, 833

Delfosse, X., Forveille, T., Beuzit, J.-L., et al. 1999, A\&A, 344, 897

Dent, W. R. F., Thi, W. F., Kamp, I., et al. 2013, PASP, 125, 477

Donati, J.-F., Gregory, S. G., Montmerle, T., et al. 2011, MNRAS, 417, 1747

Eisenbeiss, T., Ammler-von Eiff, M., Roell, T., et al. 2013, A\&A, 556, A53

Elliott, P., Bayo, A., Melo, C. H. F., et al. 2014, A\&A, 568, A26

Elliott, P., Huélamo, N., Bouy, H., et al. 2015, A\&A, 580, A88

Epchtein, N., de Batz, B., Capoani, L. et al. 1997, The Messenger, 87, 27

Evans, D. W., Irwin, M. J., \& Helmer, L. 2002, A\&A, 395, 347

Faherty, J. K., Burgasser, A. J., Walter, F. M., et al. 2012, ApJ, 752, 56

Gagné, J., Lafrenière, D., Doyon, R., Malo, L., \& Artigau, É. 2014, ApJ, 783, 121

Gagné, J., Lafrenière, D., Doyon, R., Malo, L., \& Artigau, É. 2015, ApJ, 798, 73

Girard, T. M., van Altena, W. F., Zacharias, N., et al. 2011, AJ, 142, 15

Gontcharov, G. A. 2006, Astron. Lett., 32, 759

Haakonsen, C. B., \& Rutledge, R. E. 2009, ApJS, 184, 138

Hambly, N. C., MacGillivray, H. T., Read, M. A., et al. 2001, MNRAS, 326, 1279

Høg, E., Fabricius, C., Makarov, V. V., et al. 2000, A\&A, 355, L27 
Holmberg, J., Nordström, B., \& Andersen, J. 2009, A\&A, 501, 941 Janson, M., Hormuth, F., Bergfors, C., et al. 2012, ApJ, 754, 44 Janson, M., Bergfors, C., Brandner, W., et al. 2014, ApJS, 214, 17 Jayawardhana, R., \& Brandeker, A. 2001, ApJ, 561, L111 Jenkins, J. S., Pavlenko, Y. V., Ivanyuk, O., et al. 2012, MNRAS, 420, 3587 Jiang, Y.-F., \& Tremaine, S. 2010, MNRAS, 401, 977

Kaplan, D. L., Gaensler, B. M., Kulkarni, S. R., \& Slane, P. O. 2006, ApJS, 163, 344

Kasper, M., Apai, D., Janson, M., \& Brandner, W. 2007, A\&A, 472, 321

Kastner, J. H., Sacco, G. G., Montez, R., et al. 2011, ApJ, 740, L17

Kiss, L. L., Moór, A., Szalai, T., et al. 2011, MNRAS, 411, 117

Kordopatis, G., Gilmore, G., Steinmetz, M., et al. 2013, AJ, 146, 134

Kraus, A. L., Shkolnik, E. L., Allers, K. N., \& Liu, M. C. 2014, AJ, 147, 146

Lafrenière, D., Doyon, R., Marois, C., et al. 2007, ApJ, 670, 1367

Lagrange, A.-M., Gratadour, D., Chauvin, G., et al. 2009, A\&A, 493, L21

Lagrange, A.-M., Bonnefoy, M., Chauvin, G., et al. 2010, Science, 329, 57

Lasker, B. M., Lattanzi, M. G., McLean, B. J., et al. 2008, AJ, 136, 735

Law, N. M., Dhital, S., Kraus, A., Stassun, K. G., \& West, A. A. 2010, ApJ, 720, 1727

Lépine, S., \& Gaidos, E. 2011, AJ, 142, 138

Lépine, S., \& Simon, M. 2009, AJ, 137, 3632

Li, T., Marshall, J. L., Lépine, S., Williams, P., \& Chavez, J. 2014, AJ, 148, 60

Lissauer, J. J. 2007, ApJ, 660, L149

López-Santiago, J., Montes, D., Crespo-Chacón, I., \& Fernández-Figueroa, M. J. 2006, ApJ, 643, 1160

Luger, R., Barnes, R., Lopez, E., et al. 2015, Astrobiology, 15, 57

Luhman, K. L., Mamajek, E. E., Allen, P. R., \& Cruz, K. L. 2009, ApJ, 703, 399

Luyten, W. J. 1941, Bruce proper motion survey (Minneapolis: Univ. of Minnesota Press)

Luyten, W. J. 1979, New Luyten catalogue of stars with proper motions larger than two tenths of an arcsecond and first supplement (Minneapolis)

Macintosh, B., Graham, J. R., Barman, T., et al. 2015, Science, 350, 64

Makarov, V. V. 2007, ApJS, 169, 105

Males, J. R., Close, L. M., Morzinski, K. M., et al. 2014, ApJ, 786, 32

Malo, L., Doyon, R., Lafrenière, D., et al. 2013, ApJ, 762, 88

Malo, L., Artigau, É., Doyon, R., et al. 2014a, ApJ, 788, 81

Malo, L., Doyon, R., Feiden, G. A., et al. 2014b, ApJ, 792, 37

Mamajek, E. E., \& Bell, C. P. M. 2014, MNRAS, 445, 2169

Marks, M., Janson, M., Kroupa, P., Leigh, N., \& Thies, I. 2015, MNRAS, 452, 1014

Mason, B. D., Wycoff, G. L., Hartkopf, W. I., Douglass, G. G., \& Worley, C. E. 2001, AJ, 122, 3466

Mason, B. D., Wycoff, G. L., Hartkopf, W. I., Douglass, G. G., \& Worley, C. E. 2015, VizieR on-line catalogue, B/WDS

McCarthy, K., \& White, R. J. 2012, AJ, 143, 134

Messina, S., Monard, B., Biazzo, K., Melo, C. H. F., \& Frasca, A. 2014, A\&A, 570, A19

Metchev, S. A., Eisner, J. A., Hillenbrand, L. A., \& Wolf, S. 2005, ApJ, 622, 451

Monet, D. G., Canzian, B., Harris, H. et al. 1998, VizieR on-line catalogue: $\mathrm{I} / 243$

Monet, D. G., Levine, S. E., Canzian, B., et al. 2003, AJ, 125, 984

Montes, D., López-Santiago, J., Gálvez, M. C., et al. 2001, MNRAS, 328, 45

Montet, B. T., Bowler, B. P., Shkolnik, E. L., et al. 2015, ApJ, in press, [arXiv: 1508.05945]

Moór, A., Ábrahám, P., Derekas, A., et al. 2006, ApJ, 644, 525

Moór, A., Szabó, G. M., Kiss, L. L., et al. 2013, MNRAS, 435, 1376

Mouillet, D., Larwood, J. D., Papaloizou, J. C. B., \& Lagrange, A. M. 1997, MNRAS, 292, 896

Mugrauer, M., Vogt, N., Neuhäuser, R., \& Schmidt, T. O. B. 2010, A\&A, 523, L1

Nakajima, T., \& Morino, J.-I. 2012, AJ, 143, 2

Nakajima, T., Morino, J.-I., \& Fukagawa, M. 2010, AJ, 140, 713

Neuhäuser, R., Guenther, E., Mugrauer, M., Ott, T., \& Eckart, A. 2002, A\&A, 395,877

Neuhäuser, R., Guenther, E. W., Alves, J., et al. 2003, Astron. Nachr., 324, 535

Neuhäuser, R., Ginski, C., Schmidt, T. O. B., \& Mugrauer, M. 2011, MNRAS, 416,1430
Newton, E. R., Charbonneau, D., Irwin, J., et al. 2014, AJ, 147, 20

Nordström, B., Mayor, M., Andersen, J., et al. 2004, A\&A, 418, 989

Ortega, V. G., de la Reza, R., Jilinski, E., \& Bazzanella, B. 2002, ApJ, 575, L75

Ortega, V. G., de la Reza, R., Jilinski, E., \& Bazzanella, B. 2004, ApJ, 609, 243

Parker, R. J., Goodwin, S. P., Kroupa, P., \& Kouwenhoven, M. B. N. 2009, MNRAS, 397, 1577

Pickles, A., \& Depagne, É. 2010, PASP, 122, 1437

Poveda, A., \& Allen, C. 2004, Rev. Mex. Astron. Astrofis. Ser. Conf., 21, 49

Quinn, D. P., Wilkinson, M. I., Irwin, M. J., et al. 2009, MNRAS, 396, L11

Rameau, J., Chauvin, G., Lagrange, A.-M., et al. 2013, A\&A, 553, A60

Ramírez, R. M., \& Kaltenegger, L. 2014, ApJ, 797, L25

Ramsay, G., \& Doyle, J. G. 2014, MNRAS, 442, 2926

Reid, I. N., Hawley, S. L., \& Gizis, J. E. 1995, AJ, 110, 1838

Reid, I. N., Cruz, K. L., \& Allen, P. R. 2007, AJ, 133, 2825

Reipurth, B., \& Mikkola, S. 2012, Nature, 492, 221

Retterer, J. M., \& King, I. R. 1982, ApJ, 254, 214

Riaz, B., Gizis, J. E., \& Harvin, J. 2006, AJ, 132, 866

Rice, E. L., Faherty, J. K., \& Cruz, K. L. 2010, ApJ, 715, L165

Riedel, A. R., Finch, C. T., Henry, T. J., et al. 2014, AJ, 147, 85

Rodríguez, D. R., Zuckerman, B., Faherty, J. K., \& Vican, L. 2014, A\&A, 567, A20

Roeser, S., Demleitner, M., \& Schilbach, E. 2010, AJ, 139, 2440

Rojas, G., Gregorio-Hetem, J., \& Hetem, A. 2008, MNRAS, 387, 1335

Rojas-Ayala, B., Covey, K. R., Muirhead, P. S., \& Lloyd, J. P. 2012, ApJ, 748, 93

Saarinen, S., \& Gilmore, G. 1989, MNRAS, 237, 311

Schlieder, J. E., Lépine, S., \& Simon, M. 2010, AJ, 140, 119

Schlieder, J. E., Lépine, S., \& Simon, M. 2012a, AJ, 143, 80

Schlieder, J. E., Lépine, S., \& Simon, M. 2012b, AJ, 144, 109

Shaya, E. J., \& Olling, R. P. 2011, ApJS, 192, 2

Shkolnik, E., Liu, M. C., \& Reid, I. N. 2009, ApJ, 699, 649

Shkolnik, E. L., Anglada-Escudé, G., Liu, M. C., et al. 2012, ApJ, 758, 56

Siess, L., Dufour, E., \& Forestini, M. 2000, A\&A, 358, 593

Skrutskie, M. F., Cutri, R. M., Stiening, R., et al. 2006, AJ, 131, 1163

Smart, R. L. 2013, VizieR on-line catalogues: I/324

Smith, B. A., \& Terrile, R. J. 1984, Science, 226, 1421

Song, I., Bessell, M. S., \& Zuckerman, B. 2002, ApJ, 581, L43

Song, I., Zuckerman, B., \& Bessell, M. S. 2003, ApJ, 599, 342

Strassmeier, K. G., \& Rice, J. B. 2000, A\&A, 360, 1019

Taylor, M. B. 2006, Astronomical Data Analysis Software and Systems XV, ASP Conf. Ser., 351, 666

Teixeira, R., Ducourant, C., Chauvin, G., et al. 2009, A\&A, 503, 281

Tian, F. 2015, Icarus, 258, 50

Tian, F., \& Ida, S. 2015, Nature Geosci., 8, 177

Tokovinin, A. 2014a, AJ, 147, 86

Tokovinin, A. 2014b, AJ, 147, 87

Tokovinin, A., \& Lépine, S. 2012, AJ, 144, 102

Tolbert, C. R. 1964, ApJ, 139, 1105

Torres, C. A. O., Quast, G. R., da Silva, L., et al. 2006, A\&A, 460, 695

Torres, C. A. O., Quast, G. R., Melo, C. H. F., \& Sterzik, M. F. 2008, in Handbook of Star Forming Regions, Volume II, 757

Urban, S. E., Corbin, T. E., Wycoff, G. L., et al. 1998, AJ, 115, 1212

van Altena, W. F., Lee, J. T., \& Hoffleit, E. D. 1995, 4th edn. (New Haven, CT: Yale University Observatory)

van Leeuwen, F. 2007, A\&A, 474, 653

Voges, W., Aschenbach, B., Boller, T., et al. 1999, A\&A, 349, 389

Wahhaj, Z., Liu, M. C., Nielsen, E. L., et al. 2013, ApJ, 773, 179

Ward-Duong, K., Patience, J., de Rosa, R. J., et al. 2015, MNRAS, 449, 2618

Wasserman, I., \& Weinberg, M. D. 1991, ApJ, 382, 149

Weinberg, M. D., Shapiro, S. L., \& Wasserman, I. 1987, ApJ, 312, 367

Yee, J. C., \& Jensen, E. L. N. 2010, ApJ, 711, 303

Yoo, J., Chanamé, J., \& Gould, A. 2004, ApJ, 601, 311

Zacharias, N., Finch, C. T., Girard, T. M., et al. 2013, AJ, 145, 44

Zuckerman, B., \& Song, I. 2004, ARA\&A, 42, 685

Zuckerman, B., Song, I., \& Webb, R. A. 2001a, ApJ, 559, 388

Zuckerman, B., Song, I., Bessell, M. S., \& Webb, R. A. 2001b, ApJ, 562, L87 


\section{Appendix A: Long tables}

Table A.1. Investigated $\beta$ Pictoris members and member candidates.

\begin{tabular}{|c|c|c|c|c|c|}
\hline $\begin{array}{l}\text { Simbad } \\
\text { name }\end{array}$ & $\begin{array}{c}\alpha \\
(\mathrm{J} 2000)\end{array}$ & $\begin{array}{c}\delta \\
(\mathrm{J} 2000)\end{array}$ & $\begin{array}{c}d \\
{[\mathrm{pc}]}\end{array}$ & $\begin{array}{l}\text { Reference for } \\
\text { distance }\end{array}$ & $\begin{array}{c}\text { Memb. } \\
\text { flag }\end{array}$ \\
\hline HD 203 & 00:06:50.08 & $-23: 06: 27.2$ & $39.39 \pm 0.59$ & van Leeuwen (2007) & 1 \\
\hline RBS 38 & $00: 17: 23.54$ & $-66: 45: 12.5$ & $39.0 \pm 2.6$ & Riedel et al. (2014) & 2 \\
\hline RX J0019.7+1951 & 00:19:43.04 & $+19: 51: 11.7$ & $59.4 \pm 7.9$ & Schlieder et al. (2012a) & 2 \\
\hline FK Psc & $00: 23: 34.68$ & $+20: 14: 28.3$ & $53 \pm 4$ & Malo et al. (2014b) & 2 \\
\hline 1RXS J002700.0+663025 & $00: 27: 02.83$ & $+66: 30: 39.0$ & $56.9 \pm 8.3$ & Schlieder et al. (2012a) & 3 \\
\hline GJ 2006 A & $00: 27: 50.23$ & $-32: 33: 06.4$ & $33.2 \pm 2.8$ & Riedel et al. (2014) & 1 \\
\hline GJ 2006 B & $00: 27: 50.35$ & $-32: 33: 23.9$ & $31.5 \pm 2.4$ & Riedel et al. (2014) & 1 \\
\hline LP 525-39 AB & $00: 32: 34.81$ & $+07: 29: 27.1$ & $41.1 \pm 4.4$ & Schlieder et al. (2012b) & 2 \\
\hline EROS-MP J0032-4405 & $00: 32: 55.84$ & $-44: 05: 05.8$ & $26.1 \pm 2.0$ & Gagné et al. (2014) & 2 \\
\hline 2MASS J00464841+0715177 & $00: 46: 48.41$ & $+07: 15: 17.7$ & $33.8_{-3.2}^{+2.8}$ & Gagné et al. (2015) & 2 \\
\hline TYC 2288-758-1 & $00: 48: 28.65$ & $+36: 32: 34.5$ & $61.4 \pm 8.4$ & Schlieder et al. (2012a) & 2 \\
\hline RX J0102.8+1857 & 01:02:50.99 & $+18: 56: 54.2$ & $40.9 \pm 4.4$ & Schlieder et al. (2012a) & 2 \\
\hline TYC 5853-1318-1 AB & 01:07:11.93 & $-19: 35: 36.2$ & 54 & McCarthy \& White (2012) & 2 \\
\hline LP 467-16 AB & 01:11:25.42 & $+15: 26: 21.5$ & $21.8 \pm 0.8$ & Riedel et al. (2014) & 1 \\
\hline $2 \mathrm{E} 327 \mathrm{AB}$ & $01: 13: 28.17$ & $-38: 21: 02.5$ & $29 \pm 2$ & Malo et al. (2014a) & 2 \\
\hline 2MASS J01294256-0823580 & $01: 29: 42.56$ & $-08: 23: 58.0$ & $32.5 \pm 3.2$ & Gagné et al. (2015) & 2 \\
\hline 1RXS J013514.2-071254 & $01: 35: 13.93$ & $-07: 12: 51.8$ & $37.9 \pm 2.4$ & Shkolnik et al. (2012) & 2 \\
\hline LP 648-20 (EX Cet B) & 01:36:55.17 & $-06: 47: 37.9$ & $24.0 \pm 0.4$ & Malo et al. (2014b) & 3 \\
\hline $\mathrm{BD}+17232 \mathrm{AB}$ & 01:37:39.39 & $+18: 35: 32.7$ & 52.6 & McCarthy \& White (2012) & 3 \\
\hline 1RXS J015255.9-632939 & $01: 52: 55.34$ & $-63: 29: 30.1$ & $23.7 \pm 2.4$ & Gagné et al. (2014) & 2 \\
\hline RBS 253 AB & 01:53:50.77 & $-14: 59: 50.3$ & $28 \pm 2$ & Malo et al. (2014a) & 2 \\
\hline [SLS2012] PYC J02017+0117N & 02:01:46.77 & $+01: 17: 16.2$ & $63.7 \pm 9.0$ & Schlieder et al. (2012a) & 2 \\
\hline [SLS2012] PYC J02017+0117S & 02:01:46.93 & $+01: 17: 06.0$ & $63.7 \pm 9.0$ & Schlieder et al. (2012a) & 2 \\
\hline HD 14082 B & $02: 17: 24.73$ & $+28: 44: 30.5$ & $27.34 \pm 4.26$ & van Leeuwen (2007) & 1 \\
\hline HD 14082 A & $02: 17: 25.27$ & $+28: 44: 42.3$ & $34.52 \pm 3.43$ & van Leeuwen (2007) & 1 \\
\hline RX J0217.9+1225 & 02:17:56.01 & $+12: 25: 26.6$ & $67.9 \pm 6.1$ & Binks \& Jeffries (2013) & 2 \\
\hline [SLS2012] PYC J02226+3055 & $02: 22: 40.83$ & $+30: 55: 16.0$ & $46.8 \pm 5.1$ & Schlieder et al. (2012a) & 2 \\
\hline LP $353-51$ & $02: 23: 26.63$ & $+22: 44: 06.9$ & $28.7 \pm 2.3$ & van Leeuwen (2007) & 1 \\
\hline HD 15115 & $02: 26: 16.25$ & $+06: 17: 33.1$ & $45.23 \pm 1.31$ & van Leeuwen (2007) & 1 \\
\hline AG Tri B & $02: 27: 28.05$ & $+30: 58: 40.5$ & $39.95 \pm 3.59$ & van Leeuwen (2007) & 1 \\
\hline AG Tri A & $02: 27: 29.25$ & $+30: 58: 24.7$ & $39.95 \pm 3.59$ & van Leeuwen (2007) & 1 \\
\hline $\mathrm{CD}-44753 \mathrm{Aa}, \mathrm{Ab}$ & 02:30:32.41 & $-43: 42: 23.3$ & 35.7 & McCarthy \& White (2012) & 3 \\
\hline EXO 0235.2-5216 & 02:36:51.71 & $-52: 03: 03.7$ & 28.7 & Elliott et al. (2014) & 3 \\
\hline $\mathrm{BD}+05378 \mathrm{AB}$ & $02: 41: 25.89$ & $+05: 59: 18.2$ & $42.03 \pm 2.65$ & van Leeuwen (2007) & 1 \\
\hline TVLM 831-154910 & 02:50:11.67 & $-01: 51: 29.5$ & $33.1 \pm 4.9$ & Gagné et al. (2015) & 2 \\
\hline DENIS J025344.4-795913 & 02:53:44.49 & $-79: 59: 13.3$ & $28.9_{-3.2}^{+2.8}$ & Gagné et al. (2015) & 2 \\
\hline TYC 1231-151-1 & 03:10:32.74 & $+21: 31: 44.3$ & $63.6 \pm 8.7$ & Schlieder et al. (2012a) & 2 \\
\hline 1RXS J031052.7+183855 & 03:10:53.57 & $+18: 38: 38.5$ & $67.5 \pm 9.9$ & Schlieder et al. (2012a) & 2 \\
\hline RX J0332.6+2843 ABC & 03:32:35.79 & $+28: 43: 55.5$ & $55 \pm 4$ & Malo et al. (2014b) & 2 \\
\hline 2MASS J03350208+2342356 & 03:35:02.09 & $+23: 42: 35.6$ & $42.4 \pm 2.3$ & Shkolnik et al. (2012) & 1 \\
\hline 1RXS J033936.7+453126 & 03:39:37.01 & $+45: 31: 16.0$ & $59.6 \pm 7.3$ & Schlieder et al. (2012a) & 2 \\
\hline 2MASS J03445673-1145126 & $03: 44: 56.73$ & $-11: 45: 12.6$ & $31.3_{-4.4}^{+4.0}$ & Gagné et al. (2015) & 2 \\
\hline HD $232862 \mathrm{AB}$ & 03:57:19.99 & $+50: 51: 18.6$ & $51.7 \pm 5.7$ & Schlieder et al. (2012a) & 2 \\
\hline 1RXS J041137.6+250413 & 04:11:36.38 & $+25: 04: 41.8$ & $64.2 \pm 9.0$ & Schlieder et al. (2012a) & 2 \\
\hline$c$ Eri A & 04:37:36.13 & $-02: 28: 24.8$ & $29.43 \pm 0.29$ & van Leeuwen (2007) & 1 \\
\hline$c$ Eri $\mathrm{Ca}, \mathrm{Cb}$ & 04:37:37.47 & $-02: 29: 28.4$ & $29.43 \pm 0.29$ & van Leeuwen (2007) & 1 \\
\hline 2MUCD 10320 & 04:43:37.61 & $+00: 02: 05.2$ & $25.7_{-2.4}^{+3.2}$ & Gagné et al. (2014) & 2 \\
\hline V962 Per & $04: 43: 56.87$ & $+37: 23: 03.3$ & $59 \pm 5$ & Malo et al. (2014b) & 2 \\
\hline LDS 5606 A & 04:48:00.86 & $+14: 39: 58.1$ & $65 \pm 6$ & Rodríguez et al. (2014) & 2 \\
\hline LDS 5606 B & 04:48:02.58 & $+14: 39: 51.6$ & $65 \pm 6$ & Rodríguez et al. (2014) & 2 \\
\hline V1005 Ori & 04:59:34.83 & $+01: 47: 00.7$ & $25.9 \pm 1.7$ & van Leeuwen (2007) & 1 \\
\hline CD-57 1054 & 05:00:47.15 & $-57: 15: 25.6$ & $26.78 \pm 0.81$ & van Leeuwen (2007) & 1 \\
\hline V1841 Ori & 05:00:49.29 & $+15: 27: 00.7$ & $53.8 \pm 7.5$ & Schlieder et al. (2012a) & 2 \\
\hline 1RXS J050156.7+010845 & 05:01:56.66 & $+01: 08: 42.9$ & $27.0 \pm 3.2$ & Schlieder et al. (2012b) & 2 \\
\hline LP 476-207 ABC & 05:01:58.81 & $+09: 58: 58.8$ & $24.6 \pm 1.3$ & Riedel et al. (2014) & 1 \\
\hline TYC 693-948-1 & 05:02:47.84 & $+12: 22: 56.4$ & $66.8 \pm 11.9$ & Schlieder et al. (2012a) & 2 \\
\hline RX J0506.2+0439 & 05:06:12.93 & $+04: 39: 27.2$ & $41.8 \pm 6.0$ & Schlieder et al. (2012a) & 2 \\
\hline BD-21 1074Ba,Bb & 05:06:49.47 & $-21: 35: 03.8$ & $19.2 \pm 0.5$ & Riedel et al. (2014) & 1 \\
\hline BD-21 1074A & 05:06:49.92 & $-21: 35: 09.2$ & $18.3 \pm 0.7$ & Riedel et al. (2014) & 1 \\
\hline 1RXS J050712.4+143024 & 05:07:11.37 & $+14: 30: 01.4$ & $51.2 \pm 7.0$ & Schlieder et al. (2012a) & 2 \\
\hline 1RXS J050827.3-210130 & 05:08:27.29 & $-21: 01: 44.4$ & $25 \pm 5$ & Malo et al. (2014b) & 2 \\
\hline 1RXS J051954.1+315944 & 05:19:53.18 & $+31: 59: 33.9$ & $48.4 \pm 5.2$ & Schlieder et al. (2012a) & 2 \\
\hline
\end{tabular}


Table A.1. continued.

\begin{tabular}{|c|c|c|c|c|c|}
\hline $\begin{array}{l}\text { Simbad } \\
\text { name }\end{array}$ & $\begin{array}{c}\alpha \\
(\mathrm{J} 2000)\end{array}$ & $\begin{array}{c}\delta \\
(\mathrm{J} 2000)\end{array}$ & $\begin{array}{c}d \\
{[\mathrm{pc}]}\end{array}$ & $\begin{array}{l}\text { Reference for } \\
\text { distance }\end{array}$ & $\begin{array}{c}\text { Memb. } \\
\text { flag }\end{array}$ \\
\hline TYC 112-917-1 & 05:20:00.29 & $+06: 13: 03.6$ & 68.5 & Elliott et al. (2014) & 2 \\
\hline 2E 1249 AB & $05: 20: 31.83$ & $+06: 16: 11.5$ & 69.7 & Elliott et al. (2014) & 2 \\
\hline CD-39 1935 & 05:22:45.69 & $-39: 17: 06.1$ & $33 \pm 6$ & Malo et al. (2013) & 2 \\
\hline 1RXS J052419.1-160117 AB & $05: 24: 19.14$ & $-16: 01: 15.3$ & $20 \pm 5$ & Malo et al. (2014b) & 2 \\
\hline AF Lep AB & 05:27:04.77 & $-11: 54: 03.3$ & $27.04 \pm 0.35$ & van Leeuwen (2007) & 1 \\
\hline 2E 1287 & 05:29:44.68 & $-32: 39: 14.2$ & $26.18 \pm 1.10$ & Riedel et al. (2014) & 2 \\
\hline V1311 Ori AB & 05:32:04.50 & $-03: 05: 29.2$ & $42 \pm 6$ & Malo et al. (2013) & 1 \\
\hline RBS 661 & $05: 33: 28.03$ & $-42: 57: 20.5$ & $16 \pm 4$ & Malo et al. (2013) & 2 \\
\hline RX J0534.0-0221 & 05:33:59.81 & $-02: 21: 32.5$ & $42 \pm 5$ & Malo et al. (2014b) & 2 \\
\hline 1RXS J054223.7-275803 & 05:42:23.87 & $-27: 58: 03.1$ & $44 \pm 9$ & Malo et al. (2013) & 2 \\
\hline$\beta$ Pic & 05:47:17.08 & $-51: 03: 59.5$ & $19.44 \pm 0.04$ & van Leeuwen (2007) & 1 \\
\hline 2MASS J06085283-2753583 & 06:08:52.83 & $-27: 53: 58.3$ & $31.269 \pm 3.55$ & Faherty et al. (2012) & 2 \\
\hline 1RXS J061313.2-274205 AB & 06:13:13.30 & $-27: 42: 05.4$ & $29.4 \pm 0.9$ & Riedel et al. (2014) & 1 \\
\hline TYC 6513-1245-1 & $06: 13: 57.75$ & $-27: 23: 55.3$ & $51 \pm 8$ & Malo et al. (2013) & 2 \\
\hline 1RXS J061610.6-132046 AB & $06: 16: 10.33$ & $-13: 20: 42.3$ & $47 \pm 7$ & Malo et al. (2013) & 2 \\
\hline AO Men & $06: 18: 28.24$ & $-72: 02: 41.6$ & $38.55 \pm 0.13$ & van Leeuwen (2007) & 1 \\
\hline 1RXS J065940.5+054541 & $06: 59: 41.57$ & $+05: 45: 40.0$ & $44.3 \pm 6.5$ & Schlieder et al. (2012a) & 2 \\
\hline LP 58-170 & $07: 23: 29.41$ & $+66: 46: 44.3$ & $139.28 \pm 42.48$ & van Leeuwen (2007) & 2 \\
\hline 1RXS J072643.1+185026 & $07: 26: 41.54$ & $+18: 50: 34.7$ & $57.7 \pm 8.1$ & Schlieder et al. (2012a) & 2 \\
\hline 1RXS J072931.4+355607 AB & 07:29:31.09 & $+35: 56: 00.4$ & $42.2 \pm 4.0$ & Schlieder et al. (2012b) & 2 \\
\hline YZ CMi AB & 07:44:40.17 & $+03: 33: 08.8$ & $5.96 \pm 0.08$ & van Leeuwen (2007) & 2 \\
\hline 2MASS J08025781-830076 & 08:02:57.81 & $-83: 30: 07.6$ & $20.5_{-20}^{+2.4}$ & Gagné et al. (2015) & 2 \\
\hline EUVE J0817-82.7 AB & $08: 17: 39.44$ & $-82: 43: 29.8$ & $27 \pm 2$ & Malo et al. (2014a) & 2 \\
\hline L 186-67 Aa,Ab & $08: 22: 47.45$ & $-57: 26: 53.0$ & $11.1 \pm 3.3$ & Lépine \& Gaidos (2011) & 3 \\
\hline 2MASS J08224748+0757171 & $08: 22: 47.49$ & $+07: 57: 17.2$ & $62.4 \pm 9.8$ & Schlieder et al. (2012b) & 2 \\
\hline [SLS2012] PYC J08290+1125 & 08:29:04.12 & $+11: 25: 05.4$ & $58.8 \pm 8.5$ & Schlieder et al. (2012a) & 2 \\
\hline HD 73018 AB & $08: 37: 39.24$ & $+41: 48: 02.3$ & $51.9 \pm 5.7$ & Schlieder et al. (2012a) & 2 \\
\hline [SLS2012] PYC J09226+7122S & $09: 22: 37.64$ & $+71: 22: 07.3$ & $65.9 \pm 9.6$ & Schlieder et al. (2012a) & 2 \\
\hline HD $82939 \mathrm{Ba}, \mathrm{Bb}$ & 09:36:15.91 & $+37: 31: 45.5$ & $33.75 \pm 2.61$ & van Leeuwen (2007) & 3 \\
\hline RX J1002.0+6651 & 10:01:59.95 & $+66: 51: 27.7$ & $38.7 \pm 4.0$ & Schlieder et al. (2012b) & 3 \\
\hline DK Leo AB & 10:14:19.18 & $+21: 04: 29.5$ & $23.08 \pm 0.96$ & van Leeuwen (2007) & 2 \\
\hline TWA $22 \mathrm{Aa}, \mathrm{Ab}$ & $10: 17: 26.89$ & $-53: 54: 26.5$ & $17.5 \pm 0.2$ & Malo et al. (2014b) & 1 \\
\hline [SLS2012] PYCJ10175+5542 & $10: 17: 31.43$ & $+55: 42: 29.4$ & $63.0 \pm 8.3$ & Schlieder et al. (2012a) & 2 \\
\hline RX J1035.9+2853 & $10: 35: 57.25$ & $+28: 53: 31.7$ & $37.8 \pm 3.9$ & Schlieder et al. (2012b) & 2 \\
\hline HD 95174 A & 10:59:38.31 & $+25: 26: 15.5$ & $22.6 \pm 2.0$ & Schlieder et al. (2012b) & 2 \\
\hline HD 95174 B & 10:59:38.68 & $+25: 26: 13.7$ & $22.6 \pm 2.0$ & Schlieder et al. (2012b) & 2 \\
\hline [SLS2012] PYC J11167+3814 & 11:16:46.09 & $+38: 14: 13.6$ & $67.6 \pm 9.6$ & Schlieder et al. (2012a) & 2 \\
\hline RBS 1043 Aa,Ab,B & $11: 51: 56.81$ & $+07: 31: 26.3$ & $33.2 \pm 2.7$ & Schlieder et al. (2012a) & 2 \\
\hline 2E 2613 & 12:11:53.09 & $+12: 49: 13.5$ & $62.7 \pm 8.2$ & Schlieder et al. (2012a) & 2 \\
\hline 1RXS J135452.3-712157 & 13:54:53.90 & $-71: 21: 47.7$ & $21 \pm 1$ & Malo et al. (2014a) & 3 \\
\hline TYC 4634-1184-1 & $14: 12: 49.93$ & $+84: 01: 31.2$ & $57.9 \pm 9.7$ & Schlieder et al. (2012a) & 2 \\
\hline MV Vir Aa,Ab,B & $14: 14: 21.36$ & $-15: 21: 21.7$ & $30.2 \pm 4.5$ & van Leeuwen (2007) & 2 \\
\hline SCR J1425-4113 AB & $14: 25: 29.13$ & $-41: 13: 32.4$ & $66.9 \pm 4.3$ & Riedel et al. (2014) & 3 \\
\hline StKM 1-1155 & $14: 25: 55.93$ & $+14: 12: 10.1$ & $51.8 \pm 7.3$ & Schlieder et al. (2012a) & 2 \\
\hline$\alpha \mathrm{Cir} \mathrm{AB}$ & $14: 42: 30.42$ & $-64: 58: 30.5$ & $16.57 \pm 0.03$ & van Leeuwen (2007) & 3 \\
\hline V343 Nor B & $15: 38: 56.79$ & $-57: 42: 19.0$ & $38.54 \pm 1.69$ & van Leeuwen (2007) & 1 \\
\hline V343 Nor A & $15: 38: 57.57$ & $-57: 42: 27.3$ & $38.54 \pm 1.69$ & van Leeuwen (2007) & 1 \\
\hline TYC 4571-1414-1 & $16: 17: 11.48$ & $+77: 33: 47.8$ & $65.0 \pm 13.5$ & Schlieder et al. (2012a) & 3 \\
\hline d Sco & 16:18:17.90 & $-28: 36: 50.5$ & $41.29 \pm 0.38$ & van Leeuwen (2007) & 1 \\
\hline 1RXS J164302.3-175418 & $16: 43: 01.28$ & $-17: 54: 27.4$ & $59.2 \pm 2.8$ & Binks \& Jeffries (2013) & 2 \\
\hline 1RXS J165719.9-534328 & $16: 57: 20.30$ & $-53: 43: 31.7$ & $51 \pm 3$ & Malo et al. (2014a) & 2 \\
\hline 1RXS J171502.4-333344 & $17: 15: 02.20$ & $-33: 33: 39.8$ & $23 \pm 1$ & Malo et al. (2014a) & 2 \\
\hline CD-27 $11535 \mathrm{Aa}, \mathrm{Ab}, \mathrm{B}$ & $17: 15: 03.61$ & $-27: 49: 39.7$ & 84.1 & Elliott et al. (2014) & 2 \\
\hline V824 Ara Aa,Ab & $17: 17: 25.51$ & $-66: 57: 03.9$ & $31.45 \pm 4.94$ & van Leeuwen (2007) & 1 \\
\hline V824 Ara B & $17: 17: 31.29$ & $-66: 57: 05.6$ & $31.45 \pm 4.94$ & van Leeuwen (2007) & 1 \\
\hline 1RXS J172919.1-501454 AB & $17: 29: 20.67$ & $-50: 14: 52.9$ & $64 \pm 5$ & Malo et al. (2014a) & 1 \\
\hline CD-54 7336 & $17: 29: 55.07$ & $-54: 15: 48.8$ & 66 & McCarthy \& White (2012) & 1 \\
\hline HD 160305 & $17: 41: 49.03$ & $-50: 43: 27.9$ & $72.46 \pm 4.57$ & van Leeuwen (2007) & 1 \\
\hline HD $161460 \mathrm{AB}$ & $17: 48: 33.74$ & $-53: 06: 43.3$ & 69.8 & Elliott et al. (2014) & 1 \\
\hline HD $164249 \mathrm{AB}$ & 18:03:03.41 & $-51: 38: 56.4$ & $48.15 \pm 1.30$ & van Leeuwen (2007) & 1 \\
\hline HD 165189 AB & 18:06:49.90 & $-43: 25: 30.8$ & $41.84 \pm 1.16$ & van Leeuwen (2007) & 1 \\
\hline V4046 Sgr AB & $18: 14: 10.48$ & $-32: 47: 34.4$ & $73 \pm 18$ & Kastner et al. (2011) & 1 \\
\hline V4046 Sgr C & 18:14:22.07 & $-32: 46: 10.1$ & $73 \pm 18$ & Kastner et al. (2011) & 1 \\
\hline 1RXS J181514.7-492755 & $18: 15: 15.64$ & $-49: 27: 47.2$ & $61 \pm 4$ & Malo et al. (2014a) & 2 \\
\hline HD 168210 & $18: 19: 52.21$ & $-29: 16: 32.8$ & $72.57 \pm 5.37$ & van Leeuwen (2007) & 1 \\
\hline
\end{tabular}


Table A.1. continued.

\begin{tabular}{|c|c|c|c|c|c|}
\hline $\begin{array}{l}\text { Simbad } \\
\text { name }\end{array}$ & $\begin{array}{c}\alpha \\
\text { (J2000) }\end{array}$ & $\begin{array}{c}\delta \\
(\mathrm{J} 2000)\end{array}$ & $\begin{array}{c}d \\
{[\mathrm{pc}]}\end{array}$ & $\begin{array}{l}\text { Reference for } \\
\text { distance }\end{array}$ & $\begin{array}{c}\text { Memb. } \\
\text { flag }\end{array}$ \\
\hline FK Ser AB & $18: 20: 22.75$ & $-10: 11: 13.6$ & $75.93 \pm 22.00$ & van Leeuwen (2007) & 3 \\
\hline 1RXS J184206.5-555426 & 18:42:06.95 & $-55: 54: 25.5$ & $54 \pm 4$ & Malo et al. (2014a) & 2 \\
\hline HD 172555 A & $18: 45: 26.91$ & $-64: 52: 16.5$ & $28.55 \pm 0.16$ & van Leeuwen (2007) & 1 \\
\hline $\mathrm{HD} 172555 \mathrm{Ba}, \mathrm{Bb}$ & $18: 45: 37.05$ & $-64: 51: 46.1$ & $29.24 \pm 0.60$ & van Leeuwen (2007) & 1 \\
\hline Smethells 20 (HD 173167 B) & $18: 46: 52.56$ & $-62: 10: 36.7$ & $54 \pm 3$ & Malo et al. (2014a) & 1 \\
\hline HD 173167 A & 18:48:06.36 & $-62: 13: 47.0$ & 52 & Holmberg et al (2009) & 2 \\
\hline 1RXS J184956.1-013402 & $18: 49: 55.44$ & $-01: 34: 08.7$ & $23 \pm 6$ & Malo et al. (2014b) & 3 \\
\hline CD-31 16041 & $18: 50: 44.48$ & $-31: 47: 47.2$ & $53 \pm 3$ & Malo et al. (2014a) & 1 \\
\hline PZ Tel Aa,Ab & 18:53:05.87 & $-50: 10: 50.0$ & $51.49 \pm 2.60$ & van Leeuwen (2007) & 1 \\
\hline TYC 6872-1011-1 & 18:58:04.15 & $-29: 53: 04.6$ & $76 \pm 5$ & Malo et al. (2014b) & 1 \\
\hline 2MASS J18580464-2953320 & 18:58:04.66 & $-29: 53: 32.2$ & $76 \pm 5$ & Malo et al. (2014b) & 1 \\
\hline 1RXS J191028.6-231934 & 19:10:28.20 & $-23: 19: 48.6$ & $67 \pm 5$ & Malo et al. (2014b) & 2 \\
\hline CD-26 13904 AB & $19: 11: 44.68$ & $-26: 04: 08.5$ & 80 & McCarthy \& White (2012) & 1 \\
\hline$\eta \mathrm{Tel} \mathrm{AB}$ & $19: 22: 51.22$ & $-54: 25: 26.3$ & $48.22 \pm 0.49$ & van Leeuwen (2007) & 1 \\
\hline$\eta \mathrm{Tel} \mathrm{C}$ & $19: 22: 58.95$ & $-54: 32: 17.1$ & $51.81 \pm 1.74$ & van Leeuwen (2007) & 1 \\
\hline 1RXS J192338.2-460631 & $19: 23: 38.20$ & $-46: 06: 31.6$ & $70 \pm 4$ & Malo et al. (2014b) & 2 \\
\hline RX J1924.5-3442 & $19: 24: 34.95$ & $-34: 42: 39.3$ & $54 \pm 3$ & Malo et al. (2014a) & 2 \\
\hline 2MASS J19395435-5216468 & 19:39:54.35 & $-52: 16: 46.8$ & $28.9_{-2.4}^{+2.8}$ & Gagné et al. (2015) & 2 \\
\hline HDE 331149 A & $19: 43: 37.90$ & $+32: 25: 12.5$ & $37.6 \pm 8.3$ & Schlieder et al. (2012a) & 2 \\
\hline 2MASS J19444417-4359015 & $19: 44: 44.17$ & $-43: 59: 01.5$ & $28.1 \pm 2.4$ & Gagné et al. (2015) & 2 \\
\hline 1RXS J195602.8-320720 AB & 19:56:02.94 & $-32: 07: 18.7$ & $55 \pm 4$ & Malo et al. (2014a) & 1 \\
\hline TYC 7443-1102-1 & 19:56:04.38 & $-32: 07: 37.6$ & $55 \pm 3$ & Malo et al. (2014a) & 1 \\
\hline 2MASS J20004841-7523070 & 20:00:48.42 & $-75: 23: 07.0$ & $33.3_{-28}^{+3.2}$ & Gagné et al. (2014) & 2 \\
\hline 1RXS J200136.9-331307 & 20:01:37.18 & $-33: 13: 14.0$ & $61 \pm 4$ & Malo et al. (2014a) & 2 \\
\hline HD 191089 & 20:09:05.22 & $-26: 13: 26.5$ & $52.22 \pm 1.23$ & van Leeuwen (2007) & 1 \\
\hline 1RXS J201001.0-280139 AB & 20:10:00.02 & $-28: 01: 41.0$ & $48.0 \pm 3.1$ & Riedel et al. (2014) & 1 \\
\hline SCR J2033-4903 & 20:33:01.86 & $-49: 03: 10.5$ & $16.3 \pm 5.0$ & Gagné et al. (2015) & 2 \\
\hline 1RXS J203336.9-255654 & $20: 33: 37.59$ & $-25: 56: 52.1$ & $48.3 \pm 3.3$ & Riedel et al. (2014) & 1 \\
\hline 2MASS J20334670-3733443 & $20: 33: 46.70$ & $-37: 33: 44.3$ & $33.8 \pm 2.8$ & Gagné et al. (2015) & 2 \\
\hline AU Mic BC (AT Mic AB) & 20:41:51.12 & $-32: 26: 07.3$ & $10.70 \pm 0.42$ & van Leeuwen (2007) & 1 \\
\hline StHA $182 \mathrm{AB}$ & $20: 43: 41.14$ & $-24: 33: 53.4$ & $28.1 \pm 3.9$ & Shkolnik et al. (2012) & 2 \\
\hline AU Mic & 20:45:09.49 & $-31: 20: 26.7$ & $9.91 \pm 0.10$ & van Leeuwen (2007) & 1 \\
\hline 2MASS J20513567+1924020 & $20: 51: 35.68$ & $+19: 24: 02.0$ & $51.0 \pm 8.0$ & Schlieder et al. (2012a) & 2 \\
\hline HD 199143 AB & $20: 55: 47.68$ & $-17: 06: 51.0$ & $45.66 \pm 1.60$ & van Leeuwen (2007) & 1 \\
\hline HD 199143 CD (AZ Cap AB) & $20: 56: 02.75$ & $-17: 10: 53.9$ & $45.66 \pm 1.60$ & van Leeuwen (2007) & 1 \\
\hline 1RXS J210736.5-130500 & 21:07:36.79 & $-13: 04: 58.2$ & $36 \pm 2$ & Malo et al. (2013) & 3 \\
\hline EUVE J2110-19.3 & $21: 10: 05.36$ & $-19: 19: 57.4$ & $32 \pm 2$ & Malo et al. (2014b) & 2 \\
\hline 2MASS J21103096-2710513 & 21:10:30.96 & $-27: 10: 51.3$ & $40 \pm 2$ & Malo et al. (2013) & 2 \\
\hline 2MASS J21103147-2710578 & $21: 10: 31.48$ & $-27: 10: 57.8$ & $41 \pm 3$ & Malo et al. (2014b) & 2 \\
\hline 2MASS J21140802-2251358 & $21: 14: 08.03$ & $-22: 51: 35.8$ & $22.1 \pm 1.6$ & Gagné et al. (2014) & 2 \\
\hline StKM 1-1877 & $21: 18: 33.76$ & $+30: 14: 34.6$ & $50.5 \pm 8.0$ & Schlieder et al. (2012b) & 2 \\
\hline V390 Pav A & $21: 21: 24.49$ & $-66: 54: 57.4$ & $30.2 \pm 1.3$ & van Leeuwen (2007) & 3 \\
\hline V390 Pav B & $21: 21: 28.72$ & $-66: 55: 06.3$ & $30.2 \pm 1.3$ & van Leeuwen (2007) & 3 \\
\hline $2 \mathrm{E} 4498 \mathrm{AB}$ & $21: 37: 40.19$ & $+01: 37: 13.7$ & $39.2 \pm 4.0$ & Schlieder et al. (2012b) & 2 \\
\hline 1RXS J214127.5+204302 & $21: 41: 26.62$ & $+20: 43: 10.7$ & $57.2 \pm 8.5$ & Schlieder et al. (2012a) & 2 \\
\hline 1RXS J215518.2-004603 & 21:55:17.41 & $-00: 45: 47.8$ & $47 \pm 4$ & Malo et al. (2013) & 2 \\
\hline RX J2155.3+5938 AB & $21: 55: 24.36$ & $+59: 38: 37.1$ & $29.9 \pm 4.1$ & Schlieder et al. (2012a) & 2 \\
\hline RX J2200.7+2715 & 22:00:41.58 & $+27: 15: 13.5$ & $44 \pm 4$ & Malo et al. (2013) & 1 \\
\hline LSPM J2240+0532 & 22:40:01.45 & $+05: 32: 16.3$ & $23.6 \pm 2.7$ & Gagné et al. (2015) & 2 \\
\hline CPD-72 2713 & $22: 42: 48.96$ & $-71: 42: 21.1$ & $37 \pm 2$ & Malo et al. (2014a) & 1 \\
\hline WW PsA A & $22: 44: 57.94$ & $-33: 15: 01.6$ & $23.34 \pm 1.97$ & van Leeuwen (2007) & 1 \\
\hline WW PsA B (TX PsA) & $22: 45: 00.05$ & $-33: 15: 25.8$ & $23.34 \pm 1.97$ & van Leeuwen (2007) & 1 \\
\hline 1RXS J225710.4+363950 & $22: 57: 11.31$ & $+36: 39: 45.2$ & $68.7 \pm 11.4$ & Schlieder et al. (2012b) & 2 \\
\hline LP 462-19 AB & $23: 17: 28.07$ & $+19: 36: 46.9$ & $12 \pm 1$ & Malo et al. (2013) & 2 \\
\hline AF Psc & $23: 31: 44.93$ & $-02: 44: 39.6$ & $29.15 \pm 2.64$ & van Altena et al. (1995) & 3 \\
\hline BD-13 6424 & $23: 32: 30.85$ & $-12: 15: 51.3$ & $28 \pm 1$ & Malo et al. (2014a) & 1 \\
\hline 2E 4766 & 23:50:06.39 & $+26: 59: 51.9$ & $24 \pm 2$ & Malo et al. (2014a) & 3 \\
\hline G $68-46$ & $23: 51: 22.28$ & $+23: 44: 20.8$ & $16 \pm 1$ & Malo et al. (2014a) & 3 \\
\hline
\end{tabular}


F. J. Alonso-Floriano et al.: Sixteen new stars and eight new wide systems in the $\beta$ Pictoris moving group

Table A.2. Unresolved or unidentified systems.

\begin{tabular}{|c|c|c|c|c|c|}
\hline $\begin{array}{l}\text { Simbad } \\
\text { name }\end{array}$ & $\begin{array}{l}\text { Discovery name } \\
\text { or reference }\end{array}$ & Status & $\begin{array}{c}\rho \\
{[\operatorname{arcsec}]}\end{array}$ & $\begin{array}{c}\theta \\
{[\operatorname{deg}]}\end{array}$ & $\begin{array}{l}\text { WDS } \\
\text { identifier }\end{array}$ \\
\hline LP 525-39 AB & MCT 1 & Physical & 0.7 & 334 & $00326+0729$ \\
\hline RBS 153 AB & BRG 3 & Physical & 0.4 & 168 & 01072-1936 \\
\hline LP 467-16 AB & BEU 2 & Physical & 0.3 & 241 & $01114+1526$ \\
\hline $2 \mathrm{E} 327 \mathrm{AB}$ & BRG 4 & Physical & 1.4 & 28 & 01135-3821 \\
\hline $\begin{array}{l}\text { LP 648-20 BC } \\
\text { LP 648-20 BD }\end{array}$ & $\begin{array}{l}\text { BRG } 5 \\
\text { BWL } 7\end{array}$ & $\begin{array}{l}\text { Visual } \\
\text { Visual }\end{array}$ & $\begin{array}{l}5.4 \\
6.7\end{array}$ & $\begin{array}{c}184 \\
23\end{array}$ & 01376-0645 \\
\hline $\mathrm{BD}+17232 \mathrm{AB}$ & COU 254 & Physical & 1.6 & 24 & $01377+1836$ \\
\hline RBS 253 AB & BRG 7 & Physical & 2.8 & 292 & $01538-1500$ \\
\hline HD 15115 AB & VIG 2 & Visual & 12.6 & 195 & $02263+0618$ \\
\hline $\mathrm{BD}+05378 \mathrm{AB}$ & Song et al. (2003) & SB1 & $\ldots$ & $\ldots$ & $\ldots$ \\
\hline $\mathrm{CD}-44753 \mathrm{Aa}, \mathrm{Ab}$ & Elliott et al. (2015) & Physical & 0.13 & 295 & $02305-4305$ \\
\hline $\begin{array}{l}\text { RX J0332.6+2843 AB } \\
\text { RX J0332.6+2843 AC }\end{array}$ & $\begin{array}{l}\text { JNN } 24 \\
\text { JNN } 24\end{array}$ & $\begin{array}{l}\text { Physical } \\
\text { Physical }\end{array}$ & $\begin{array}{l}0.1 \\
0.5\end{array}$ & $\begin{array}{l}282 \\
106\end{array}$ & $03326+2844$ \\
\hline HD $232862 \mathrm{AB}$ & COU 2357 & Physical & 0.7 & 93 & $03573+5051$ \\
\hline $\begin{array}{l}c \text { Eri } \mathrm{Ab} \\
c \text { Eri } \mathrm{Ca}, \mathrm{Cb}\end{array}$ & $\begin{array}{l}\text { Macintosh et al. (2015) } \\
\text { KAS 1 }\end{array}$ & $\begin{array}{l}\text { Physical } \\
\text { Physical }\end{array}$ & $\begin{array}{l}0.4 \\
0.3\end{array}$ & $\begin{array}{c}170 \\
18\end{array}$ & 04376-0228 \\
\hline $\begin{array}{l}\text { V1005 Ori AB } \\
\text { V1005 Ori AC } \\
\text { V1005 Ori Aa,Ab }\end{array}$ & $\begin{array}{l}\text { LAF } 33 \\
\text { LAF } 33 \\
\text { Elliott et al. (2014) }\end{array}$ & $\begin{array}{l}\text { Visual } \\
\text { Visual } \\
\text { SB1? }\end{array}$ & $\begin{array}{l}5.2 \\
7.4 \\
\cdots\end{array}$ & $\begin{array}{c}220 \\
234 \\
\ldots\end{array}$ & $04596+0147$ \\
\hline $\begin{array}{l}\text { LP 476-207 Aa,Ab } \\
\text { LP 476-207 Aa,Ab,B }\end{array}$ & $\begin{array}{l}\text { Delfosse et al. (1999) } \\
\text { HDS } 654\end{array}$ & $\begin{array}{l}\text { SB2 } \\
\text { Physical }\end{array}$ & $\begin{array}{l}\ldots \\
1.3\end{array}$ & $\ldots$ & $05020+0959$ \\
\hline BD-21 1074Ba,Bb & DON 93 & Physical & 0.8 & 321 & 05069-2135 \\
\hline $2 \mathrm{E} 1249 \mathrm{AB}$ & Elliott et al. (2015) & Physical & 0.42 & 235 & $\ldots$ \\
\hline 1RXS J052419.1-160117 AB & BRG 21 & Physical & 0.6 & 68 & $05243-1601$ \\
\hline AF Lep AB & Nordström et al. (2004) & SB2 & $\ldots$ & $\ldots$ & $\ldots$ \\
\hline V1311 Ori AB & JNN 39 & Physical & 0.2 & 56 & $05321-0305$ \\
\hline 1RXS J061313.2-274205 AB & TSN 2 & Physical & 0.1 & 215 & 06132-2742 \\
\hline 1RXS J061610.6-132046 AB & BRG 22 & Physical & 0.2 & 167 & 06162-1232 \\
\hline 1RXS J072931.4+355607 AB & JNN 57 & Physical & 0.2 & 253 & $07295+3556$ \\
\hline YZ CMi AB & LAF 36 & Visual & 8.8 & 114 & $07447+0333$ \\
\hline EUVE J0817-82.7 AB & CVN 22 & Physical & 0.6 & 353 & $08177-8243$ \\
\hline L 186-67 Aa,Ab & BRG27 & Physical & 0.8 & 150 & $08228-5727$ \\
\hline HD 73018 AB & STF 1244 & Physical & 3.9 & 0 & $08377+4148$ \\
\hline HD $82939 \mathrm{Ba}, \mathrm{Bb}$ & Schlieder et al. (2012b) & SB2 & $\ldots$ & $\ldots$ & $\ldots$ \\
\hline DK Leo AB & Shkolnik et al. (2012) & SB1 & $\ldots$ & $\ldots$ & $\ldots$ \\
\hline $\begin{array}{l}\text { TWA } 22 \mathrm{Aa}, \mathrm{Ab} \\
\text { TWA } 22 \mathrm{Aa}, \mathrm{Ab}-\mathrm{Zd}\end{array}$ & $\begin{array}{l}\text { CVN } 16 \\
\text { CVN } 16\end{array}$ & $\begin{array}{l}\text { Physical } \\
\text { Visual }\end{array}$ & $\begin{array}{c}0.1 \\
3.5-16.8\end{array}$ & $\begin{array}{l}15 \\
\ldots\end{array}$ & $10174-5354$ \\
\hline $\begin{array}{l}\text { RBS } 1043 \mathrm{Aa}, \mathrm{Ab} \\
\text { RBS } 1043 \mathrm{Aa}, \mathrm{Ab}, \mathrm{B}\end{array}$ & $\begin{array}{l}\text { Bowler et al. (2015) } \\
\text { Bowler et al. (2015) }\end{array}$ & $\begin{array}{l}\text { SB2 } \\
\text { Physical }\end{array}$ & $\begin{array}{l}\ldots \\
0.5\end{array}$ & $\ldots$ & $\cdots$ \\
\hline $\begin{array}{l}\text { MV Vir } \mathrm{Aa}, \mathrm{Ab} \\
\text { MV Vir } \mathrm{Aa}, \mathrm{Ab}, \mathrm{B}\end{array}$ & $\begin{array}{l}\text { CVN } 25 \\
\text { RST } 3869\end{array}$ & $\begin{array}{l}\text { Physical } \\
\text { Physical }\end{array}$ & $\begin{array}{l}0.3 \\
1.1\end{array}$ & $\begin{array}{c}257 \\
62\end{array}$ & $14144-1521$ \\
\hline SCR J1425-4113 AB & Riedel et al. (2014) & Physical & 0.6 & 282 & $\ldots$ \\
\hline$\alpha$ Cir $\mathrm{AB}$ & DUN 166 & Physical & 15.4 & 226 & $14425-6459$ \\
\hline $\begin{array}{l}\text { V343 Nor AB } \\
\text { V343 Nor A-Zd } \\
\text { V343 Nor B-T }\end{array}$ & $\begin{array}{l}\text { SKF1501 } \\
\text { VGT } 4 \\
\text { CVN } 52\end{array}$ & $\begin{array}{l}\text { Physical } \\
\text { Visual } \\
\text { Visual }\end{array}$ & $\begin{array}{l}10.2 \\
2.2-7.5 \\
1.4-7.9\end{array}$ & $\begin{array}{c}325 \\
\ldots \\
\cdots\end{array}$ & $15390-5742$ \\
\hline $\begin{array}{l}\text { CD-27 } 11535 \mathrm{Aa}, \mathrm{Ab} \\
\mathrm{CD}-2711535 \mathrm{Aa}, \mathrm{Ab}, \mathrm{B}\end{array}$ & $\begin{array}{l}\text { Elliott et al. (2015) } \\
\text { Elliott et al. (2015) }\end{array}$ & $\begin{array}{l}\text { SB1 } \\
\text { Physical }\end{array}$ & $\begin{array}{c}\ldots \\
0.08\end{array}$ & $\begin{array}{c}\ldots \\
278\end{array}$ & $\cdots$ \\
\hline $\begin{array}{l}\text { V824 Ara AC } \\
\text { V824 Ara Aa,Ab }\end{array}$ & $\begin{array}{l}\text { CVN55 } \\
\text { Strassmeier \& Rice (2000) }\end{array}$ & $\begin{array}{l}\text { Visual } \\
\text { SB2 }\end{array}$ & $\begin{array}{l}7.9 \\
\ldots\end{array}$ & $\begin{array}{c}120 \\
\ldots\end{array}$ & $\begin{array}{l}17174-6657 \\
\ldots\end{array}$ \\
\hline 1RXS J172919.1-501454 AB & Elliott et al. (2015) & Physical & 0.73 & 20 & $\ldots$ \\
\hline HD $161460 \mathrm{AB}$ & CVN 29 & Physical & 0.1 & 233 & $17486-5307$ \\
\hline $\begin{array}{l}\text { HD } 164249 \text { AB } \\
\text { HD } 164249 \text { A-J }\end{array}$ & $\begin{array}{l}\text { SKF } 1420 \\
\text { CVN57 }\end{array}$ & $\begin{array}{l}\text { Physical } \\
\text { Visual }\end{array}$ & $\begin{array}{c}6.7 \\
7.4-14.8 \\
\end{array}$ & $\begin{array}{l}89 \\
\ldots\end{array}$ & $18031-5139$ \\
\hline
\end{tabular}


Table A.2. continued.

\begin{tabular}{lllccc}
\hline \hline $\begin{array}{l}\text { Simbad } \\
\text { name }\end{array}$ & $\begin{array}{l}\text { Discovery name } \\
\text { or reference }\end{array}$ & Status & $\begin{array}{c}\rho \\
{[\operatorname{arcsec}]}\end{array}$ & $\begin{array}{c}\theta \\
{[\mathrm{deg}]}\end{array}$ & $\begin{array}{l}\text { WDS } \\
\text { identifier }\end{array}$ \\
\hline HD 165189 AB & HJ 5014 & Physical & 1.8 & 4 & $18068-4325$ \\
V4046 Sgr AB & Torres et al. (2008) & SB2 & $\ldots$ & $\ldots$ & $\ldots$ \\
FK Ser AB & HER 2 & Physical & 1.0 & 17 & $18204-1011$ \\
HD 172555 AC & CVN 59 & Visual & 7.4 & 319 & $18454-6452$ \\
HD 172555 Ba,Bb & BIL 4 & Physical & 0.2 & 95 & \\
CD-31 16041 A-J & CVN 60 & Visual & $1.9-6.9$ & $\ldots$ & $18507-3148$ \\
PZ Tel Aa,Ab & MUG 10 & Physical & 0.4 & 60 & $18531-5011$ \\
PZ Tel Aa,Ab-E & CVN 61 & Visual & $4.0-10.7$ & $\ldots$ & \\
CD-26 13904 AB & RST 2094 & Physical & 1.1 & 49 & $19117-2604$ \\
$\eta$ Tel AB & LWR 3 & Physical & 4.2 & 170 & $19229-5425$ \\
$\eta$ Tel CD & CVN 63 3isual & 4.8 & 251 & $19230-5432$ \\
$\eta$ Tel CE & CVN 63 & Visual & 5.8 & 275 & \\
1RXS J195602.8-320720 AB & Elliott et al. (2014) & SB2 & $\ldots$ & $\ldots$ & $\ldots$ \\
HD 191089 A-C & MET 88 & Visual & $10.4-10.8$ & $\ldots$ & $20091-2613$ \\
HD 191089 A-H & VIG 19 & Visual & $4.8-12.3$ & $\ldots$ & \\
1RXS J201001.0-280139 AB & BRG 30 & Physical & 0.7 & 283 & $20100-2802$ \\
StHA 182 AB & Shkolnik et al. (2012) & Physical & 1.5 & 217 & $\ldots$ \\
AU Mic BC (AT Mic AB) & LDS 720 & Physical & 2.3 & 153 & $20452-3120$ \\
HD 199143 AB & JAY 1 & Physical & 1.1 & 324 & $20558-1707$ \\
HD 199143 CD (AZ Cap AB) & JAY 2 & Physical & 2.2 & 140 & \\
2E 4498 AB & JNN 291 & Physical & 0.4 & 341 & $21376+0137$ \\
RX J2155.3+5938 AB & JNN 292 & Phsyical & 0.2 & 102 & $21554+5938$ \\
LP 462-19 AB & BEU 23 & Physical & 0.1 & 220 & $23175+1937$ \\
\hline
\end{tabular}


F. J. Alonso-Floriano et al.: Sixteen new stars and eight new wide systems in the $\beta$ Pictoris moving group

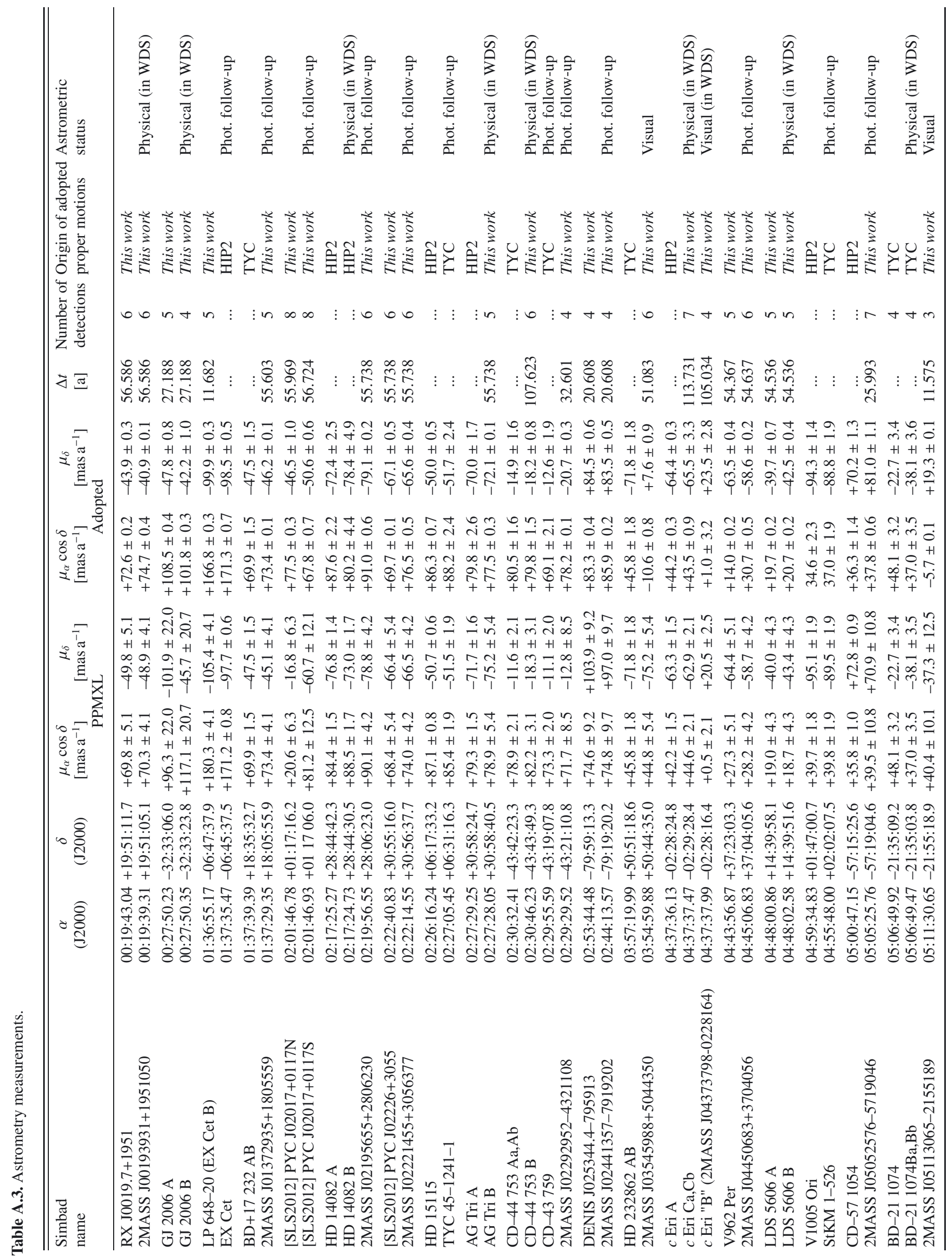




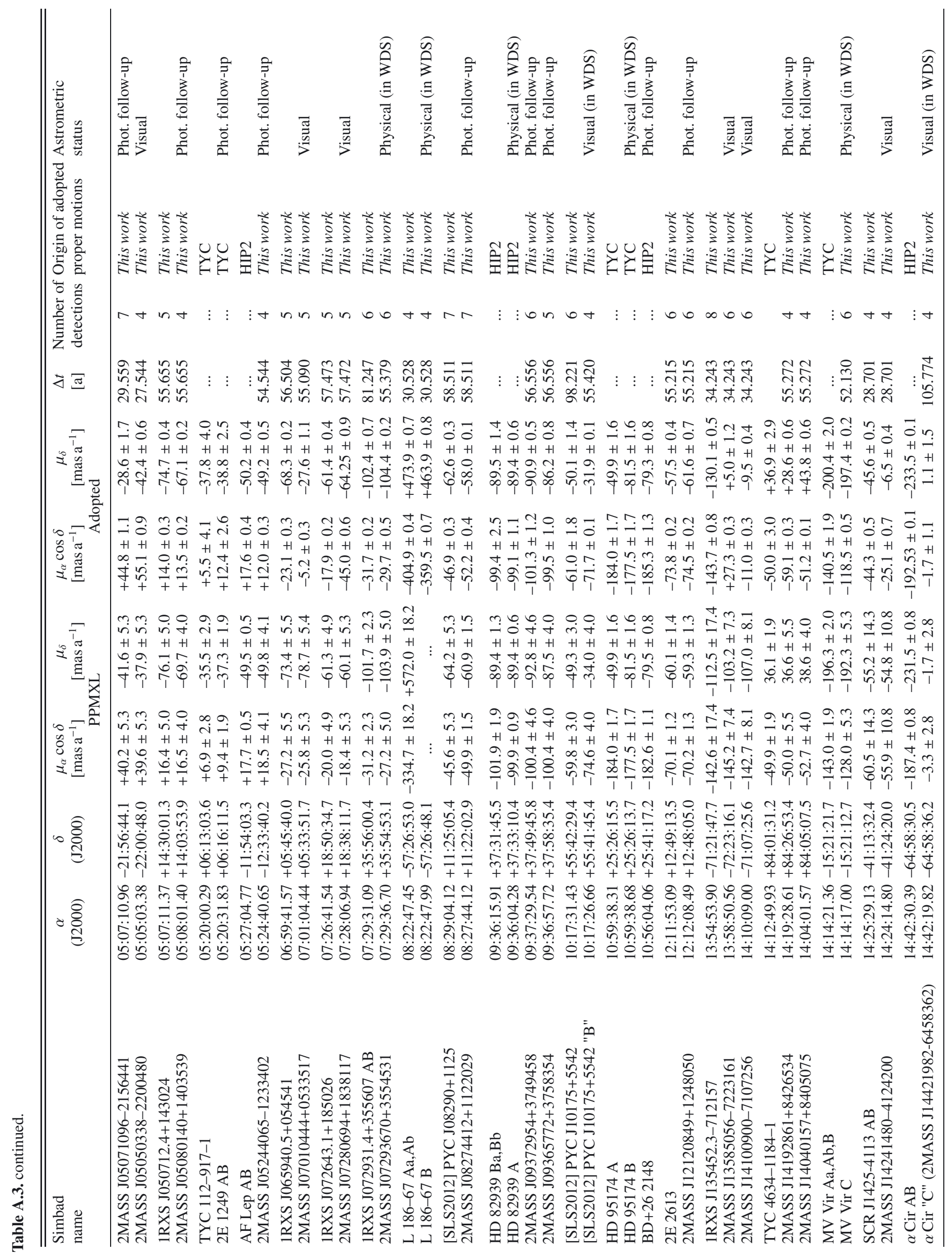


F. J. Alonso-Floriano et al.: Sixteen new stars and eight new wide systems in the $\beta$ Pictoris moving group

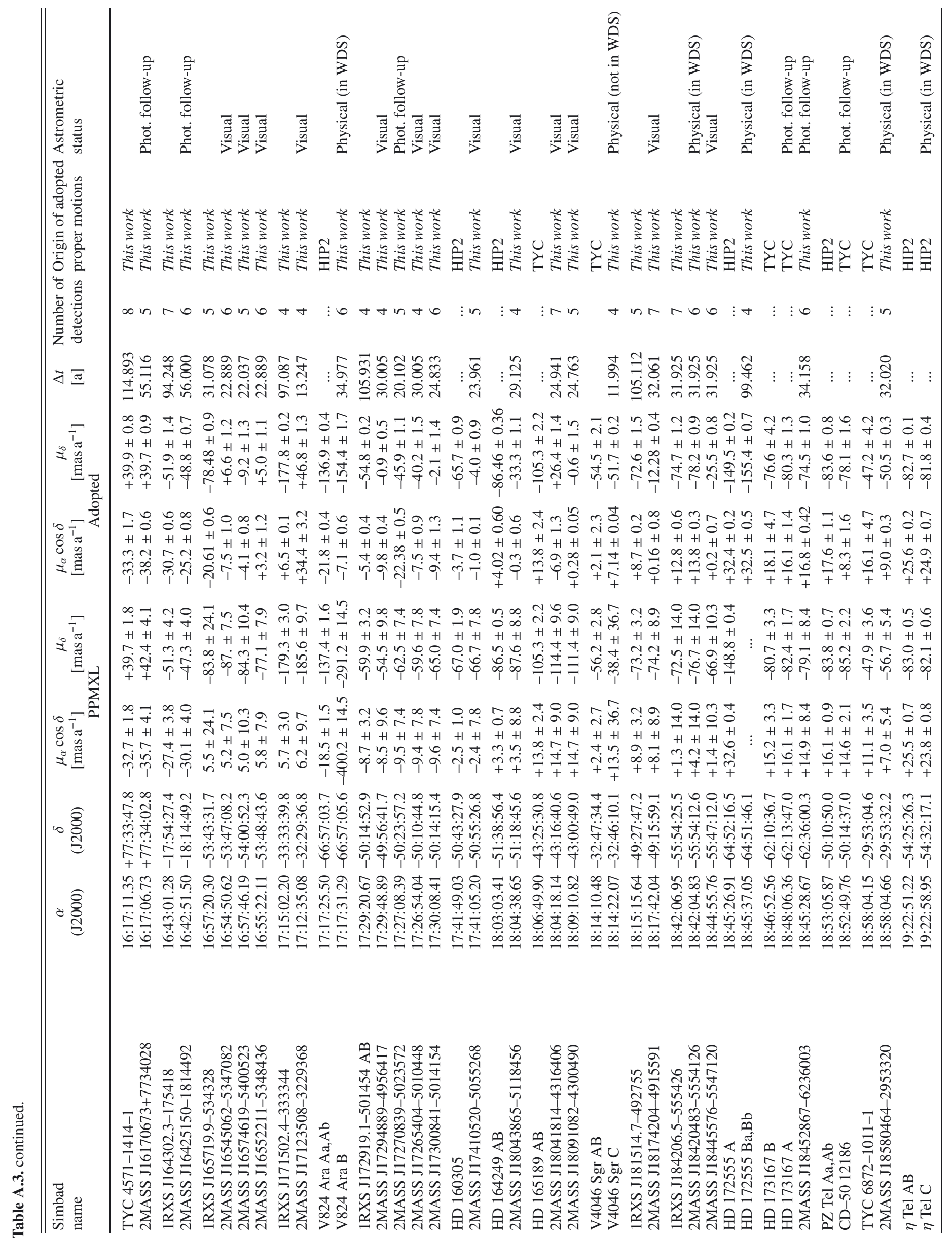




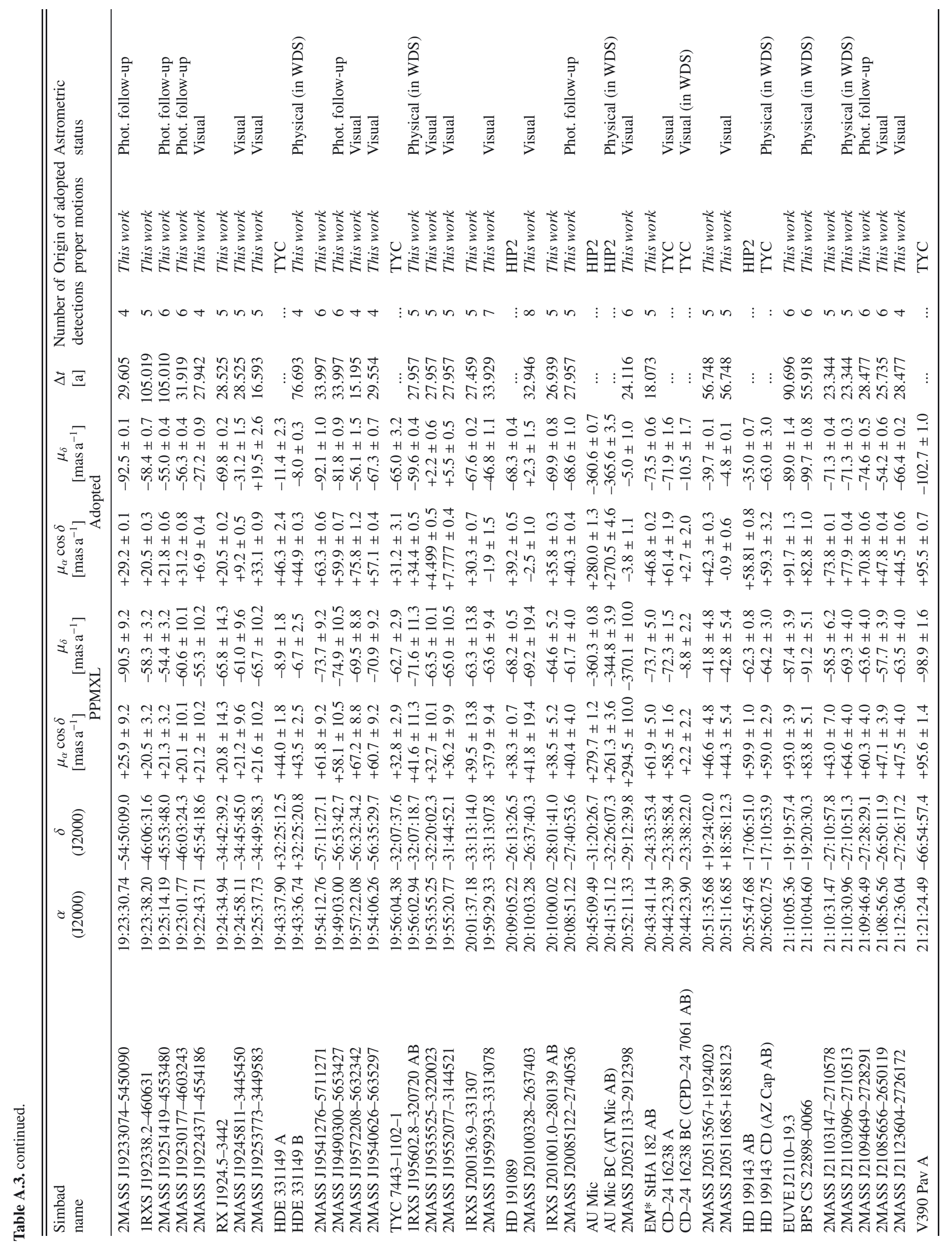


F. J. Alonso-Floriano et al.: Sixteen new stars and eight new wide systems in the $\beta$ Pictoris moving group

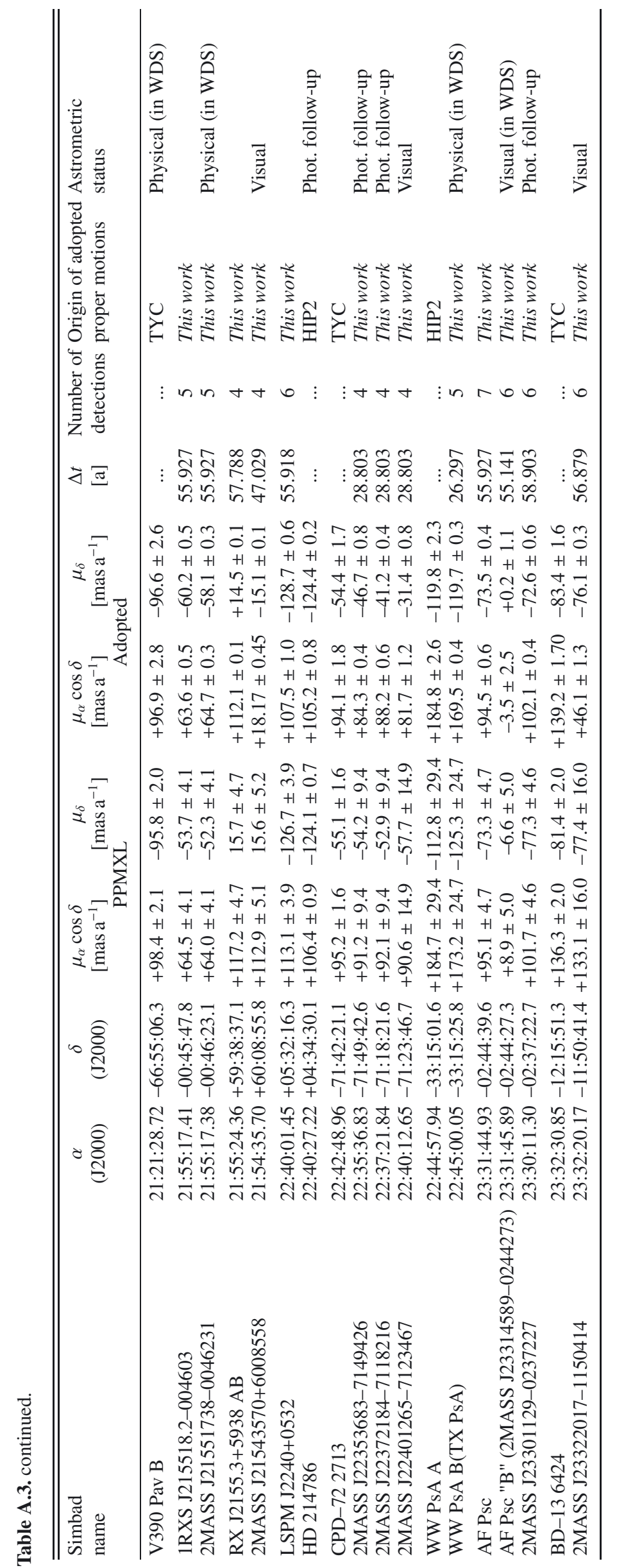


A\&A 583, A85 (2015)

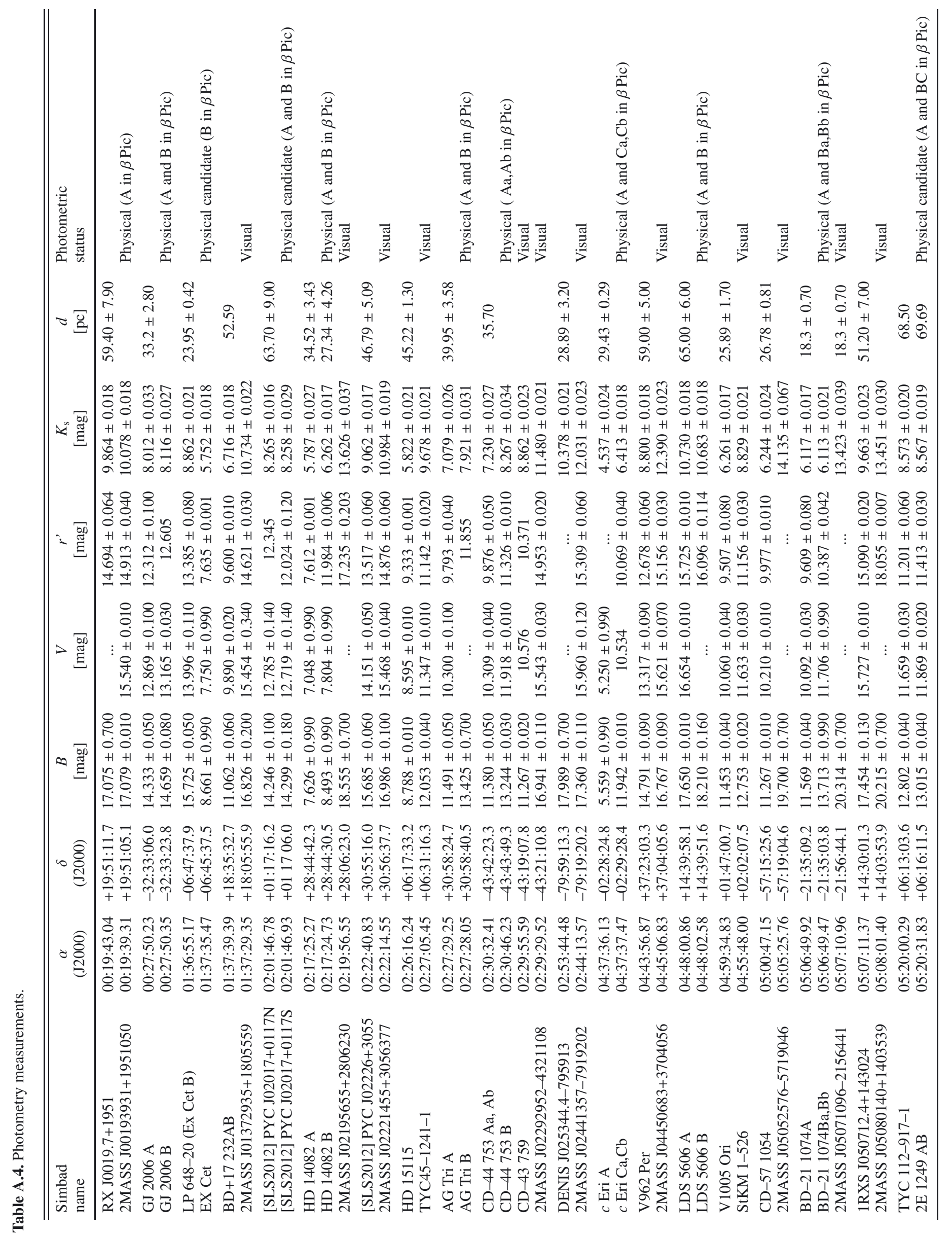


F. J. Alonso-Floriano et al.: Sixteen new stars and eight new wide systems in the $\beta$ Pictoris moving group

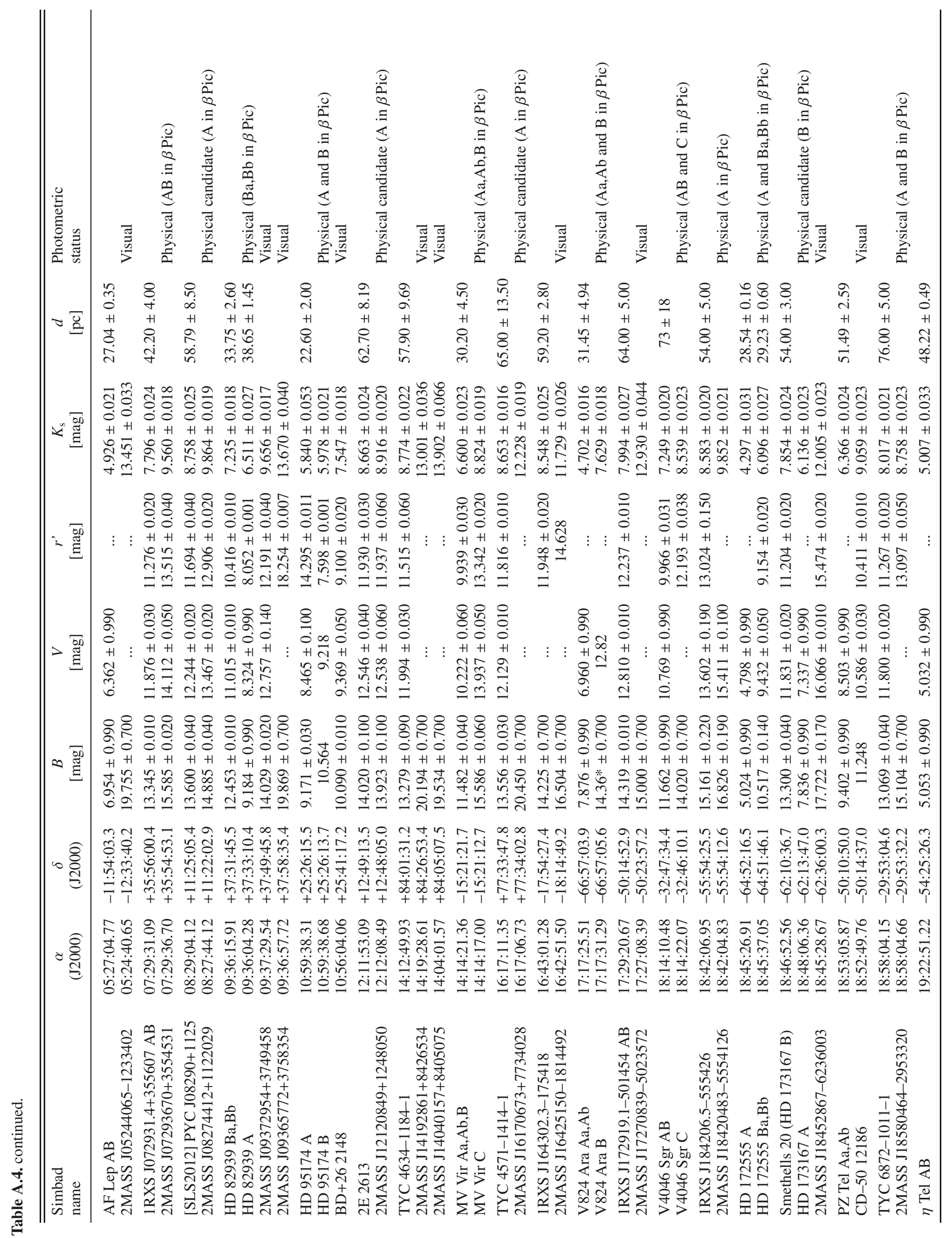




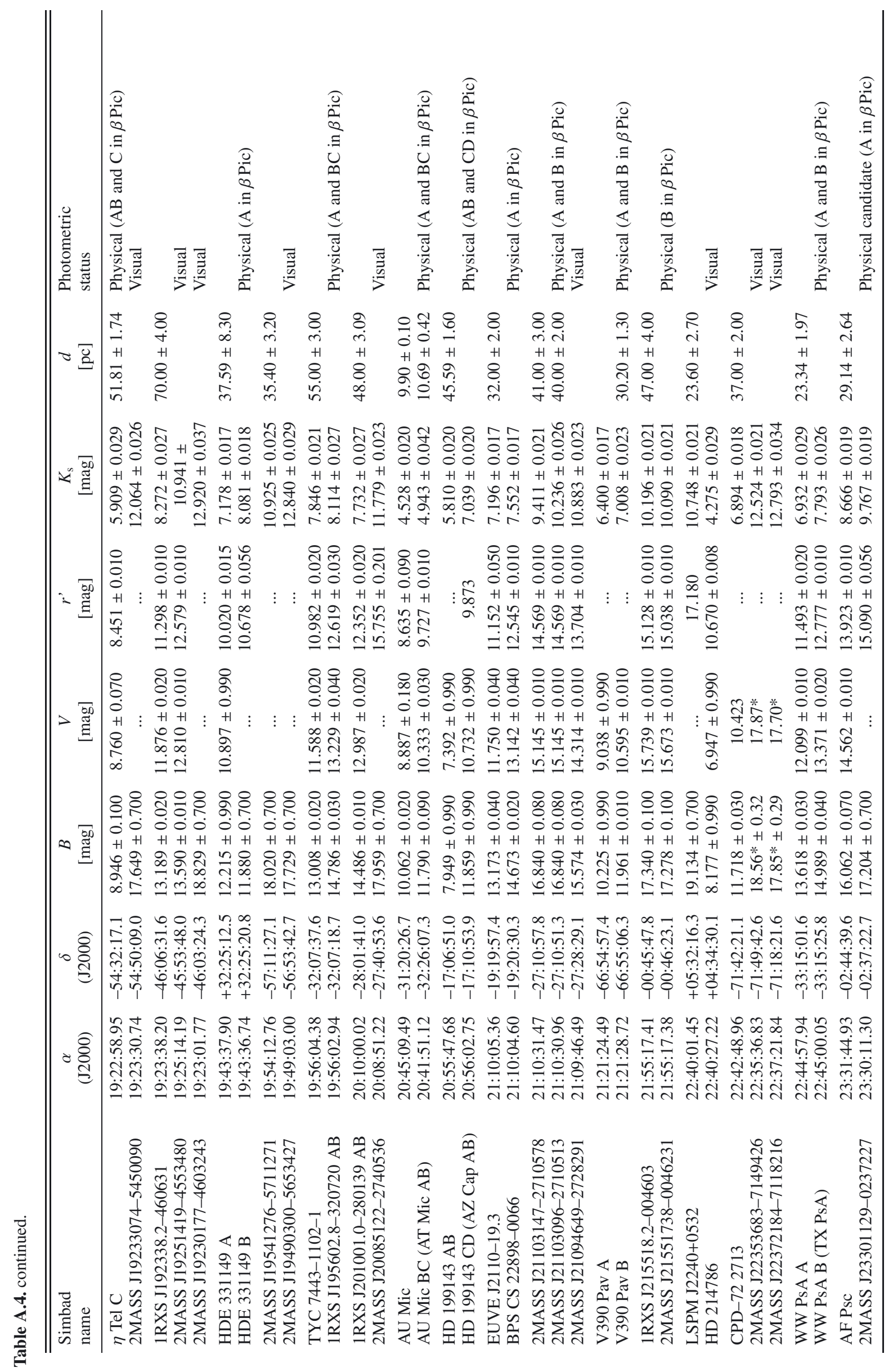




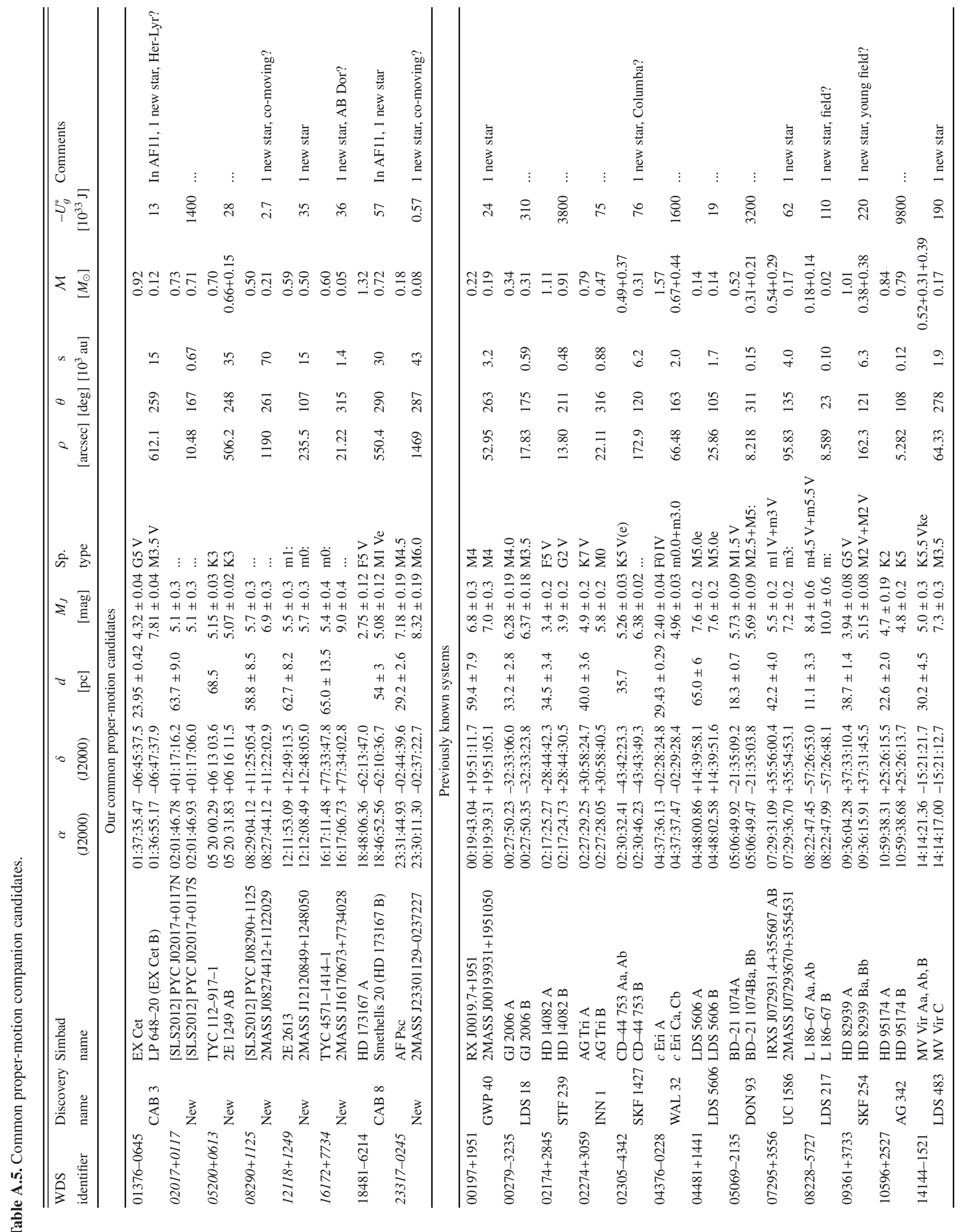




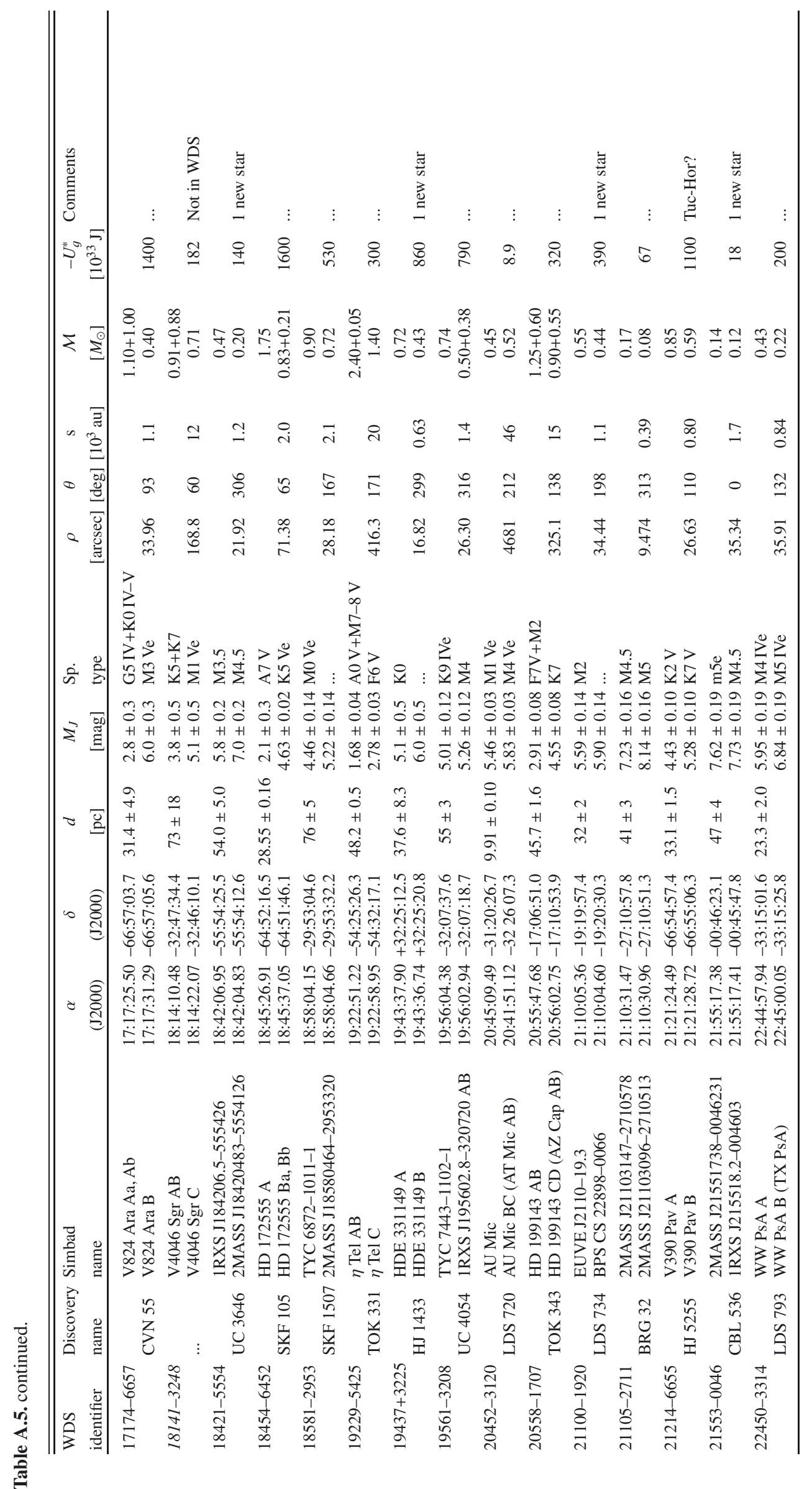

